\title{
Warm inflation with non-comoving scalar field and radiation fluid
}

\author{
Tiberiu Harko ${ }^{1,2,3, a}$, Haidar Sheikhahmadi ${ }^{4,5, b}$ \\ ${ }_{1}$ Astronomical Observatory, 19 Ciresilor Street, 400487 Cluj-Napoca, Romania \\ 2 Department of Physics, Babes-Bolyai University, Kogalniceanu Street, 400084 Cluj-Napoca, Romania \\ ${ }^{3}$ School of Physics, Sun Yat-Sen University, Guangzhou 510275, People's Republic of China \\ ${ }^{4}$ Center for Space Research, North-West University, Mafikeng, South Africa \\ ${ }^{5}$ School of Astronomy, Institute for Research in Fundamental Sciences (IPM), P.O. Box 19395-5531, Tehran, Iran
}

Received: 27 September 2020 / Accepted: 7 February 2021 / Published online: 17 February 2021

(C) The Author(s) 2021

\begin{abstract}
We consider a warm inflationary scenario in which the two major fluid components of the early Universe, the scalar field and the radiation fluid, evolve with distinct four-velocities. This cosmological configuration is equivalent to a single anisotropic fluid, expanding with a fourvelocity that is a combination of the two fluid four-velocities. Due to the presence of anisotropies the overall cosmological evolution is also anisotropic. We obtain the gravitational field equations of the non-comoving scalar field-radiation mixture for a Bianchi Type I geometry. By assuming the decay of the scalar field, accompanied by a corresponding radiation generation, we formulate the basic equations of the warm inflationary model in the presence of two noncomoving components. By adopting the slow-roll approximation the theoretical predictions of the warm inflationary scenario with non-comoving scalar field and radiation fluid are compared in detail with the observational data obtained by the Planck satellite in both weak dissipation and strong dissipation limits, and constraints on the free parameters of the model are obtained. The functional forms of the scalar field potentials compatible with the non-comoving nature of warm inflation are also obtained.
\end{abstract}

\section{Contents}

1 Introduction

2 Anisotropic fluid description of the non-comoving scalar-field-radiation cosmological models . . . . .

2.1 Four-velocities and energy-momentum tensors

2.2 Single anisotropic fluid representation of twofluid systems $\ldots \ldots \ldots \ldots$

\footnotetext{
a e-mail: tiberiu.harko@aira.astro.ro (corresponding author)

be-mails: h.sh.ahmadi@gmail.com; h.sheikhahmadi@ipm.ir
}

3 Warm inflation with non-comoving scalar field and radiation ................. 9

3.1 Brief review of the warm inflationary scenario . 9

3.2 Gravitational field equations of the two noncomoving fluid cosmological model . . . . 10

3.2.1 Field equations for a Bianchi Type I geometry 10

3.3 Warm inflation with non-comoving cosmological fluids . . . . . . . . . . . . . 11

4 The slow-roll approximation . . . . . . . . . . 12

4.1 Evolution equations for non-comoving warm inflation . . . . . . . . . . . 12

4.2 Weak dissipative regime . . . . . . . . . 13

4.2.1 Weak dissipative regime with power-law scale factor . . . . . . . . . . 14

4.2.2 Weak dissipative regime with generalized de Sitter scale factor . . . . . . . . . 15

4.3 Strong dissipative regime . . . . . . . . 16 4.3.1 Strong dissipative regime with power-law scale factor . . . . . . . . . 17

4.3.2 Strong dissipative regime with generalized de Sitter scale factor . . . . . . . . 19

5 Discussions and final remarks . . . . . . . . 20

References . . . . . . . . . . . . . . . . 22

\section{Introduction}

The recently released Planck data of the 2.7 full sky survey [1-7] have shown a number of intriguing features, whose explanation will certainly require a deep change in our standard view of the Universe. The current observations have measured the properties of the Cosmic Microwave Background Radiation (CMB) to an unprecedented precision. One of the most substantial results of the nominal mission is that the best-fit Hubble constant has the value $H_{0}=67.4 \pm 1.2 \mathrm{~km} \mathrm{~s}^{-1} \mathrm{Mpc}^{-1}$, with a dark energy den- 
sity parameter $\Omega_{\Lambda}=0.686 \pm 0.020$, and a matter density parameter $\Omega_{M}=0.307 \pm 0.019$.

Generally the Planck data confirm the foundations of the $\Lambda \mathrm{CDM}$ ( $\Lambda$ Cold Dark Matter) model. According to this model, the main material composition of the Universe can be reduced to two components: dark energy and dark matter, respectively, [8,9]. The observed late-time cosmic acceleration of the Universe [10-13] can be successfully explained by introducing either a fundamental cosmological constant $\Lambda$ [14], which would represent an intrinsic curvature of spacetime, or a dark energy, a hypothetical fluid component in the form of a zero-point-energy that pervades the whole Universe, which would mimic a cosmological constant (at least during the late stage of the cosmological evolution) $[8,9,15-$ 19]. Currently one of the main dark energy scenarios is based on the so-called quintessence [20-24], where dark energy corresponds to a scalar particle $\phi$. For other proposals for dark energy, in which the dynamical equation of state is realized by a scalar field, one can refer to k-essence [25-27], tachyon [28,29, 175], phantom [30-32], quintom[33-35], chameleon [36-41], and Chaplygin gas [42,43] models, which have also been investigated. Modifying gravity at galactic or astrophysical scales, or considering extra-dimensional cosmological models, can also lead to an explanation of the late acceleration of the Universe [44-51]. Scalar fields or other long range coherent fields coupled to gravity have also been considered as potential dark matter candidates [52-55].

The basic paradigm of the present day cosmology about the very early Universe is represented by the inflationary theory, initiated in [56]. The basic idea of inflation is the presence in the early Universe of a scalar field $\phi$, with a selfinteraction potential $V(\phi)$, and with a corresponding energy density $\rho_{\phi}$, and pressure $p_{\mid p h i}$, respectively [57]. The early inflationary models were based on the assumption that the scalar field potential reaches a local minimum at $\phi=0$, due to the supercooling after a phase transition. Subsequently the Universe enters an exponentially expanding, de Sitter type phase. However, in this initial theoretical model, the so-called old inflationary scenario, there is no graceful exit from the de Sitter accelerating, inflationary phase. Several inflationary models, with the explicit objective of solving the graceful exit problem, have been proposed, including the new and the chaotic inflationary models [58-61]. Note that each of these theories has its own and specific theoretical problems. Recent reviews of different aspects of the inflationary and of cosmology have been presented in $[62,63]$.

During inflation the exponential growth of the scale factor of the Universe leads to a homogeneous, isotropic but matterless Universe, in which all initial components have diminished to near zero. Hence, in order to explain the present composition of the Universe it is necessary for radiation and the basic elementary particles to be created at the end of inflation, in a cosmological epoch known as reheating. Dur- ing this period matter (mostly in the form of radiation) was created through the transfer of energy from the inflationary scalar field to the elementary particles. The reheating model was initially developed in the framework of the new inflationary scenario [58], and later on extended in [64-66]. The basic idea of reheating can be formulated as follows. After the de Sitter type accelerated expansion of the Universe, the scalar field driving inflation reaches its minimum value. Then it starts to oscillate around the minimum of the potential, and subsequently it disintegrates into matter, in the form of a radiation (photon) gas, and of some Standard Model elementary particles. Due to the interaction of these components, the early Universe finally reaches a thermal equilibrium state characterized by a temperature $T$.

One of the approaches extensively used to investigate reheating is the phenomenological model introduced in [59]. The basic idea of this formalism is the introduction of a particular decay term in the Klein-Gordon equation describing the evolution of the scalar field. This term is also included as a growth term in the balance equation for the energy density of the newly created particles. If reasonably chosen, the loss/gain term can describe the reheating process that did follow the adiabatic supercooling during the inflationary era. Hence, in this reheating model, an interacting twocomponent fluid, representing a mixture between the scalar field, and ordinary matter, can explain the chemical composition of the Universe. Hence the entire transition process between the scalar field and the ordinary matter component can be described phenomenologically once the functional form of the friction term, describing the decay of the scalar inflaton field, is given. The same term also represents the source term for the newly created matter fluid. For investigations of the diverse cosmological and physical aspects of the post-inflationary reheating dynamics see [67-78]. For detailed reviews on the post inflationary reheating phase see [79] and [80], respectively.

Despite its remarkable theoretical success the standard reheating model is plagued by a number of problems. One important question is related to the perturbative description of the decay width of the scalar field, which can describe the decay only close to the minimum of the potential. Such a description is not valid during slow-roll inflation. Another problem is that finite temperature effects can also significantly increase the rate at which the scalar field dissipates its energy to the newly created particles $[79,80]$.

On the other hand it is natural to assume that the scalar field driving inflation could have been coupled nonminimally to the other matter components present in the early Universe. Therefore the scalar field could have dissipated its energy during the accelerated expansion, thus warming up the Universe without the need of a reheating phase. This cosmological interpretation of inflation is called warm inflation, and it was initially proposed in $[81,82]$. Hence, according to 
the warm inflationary scenario, dissipative effects and particle creation processes can create a thermal bath (radiation fluid) during the accelerated expansion phase. In one of the first warm inflation models [83] it was suggested that in the inflationary scenarios the physical parameters could be randomly distributed. This hypothesis led to the development of distributed-mass models [84-88], developed in relation to string theory. Warm inflation represents presently a very active field of study, and it certainly represents an attractive alternative to the cold inflation/reheating scenarios. The cosmological evolution in the warm inflationary models has been investigated in detail in [89-138].

The Planck observational data also indicate the possible existence of some tension between the fundamental principle of the $\Lambda \mathrm{CDM}$ model and observations. For example, after combining the Planck data with the WMAP polarization data, the index of the power spectrum is found to be $n_{s}=$ $0.9603 \pm 0.0073[2,3]$, at the pivot scale $k_{0}=0.05 \mathrm{Mpc}^{-1}$. This value rules out the exact scale-invariance $\left(n_{s}=1\right)$ at more than $5 \sigma$. Moreover, the joint constraints on the tensorto-scalar ratio $r$ and $n_{s}$ are able to significantly restrain inflation models. For example, one should note that inflationary models with a power-law potential of the form $\phi^{4}$ cannot provide a legitimate number of $e$-folds (between 50-60) in the restricted space of $r-n_{s}$ at around a $2 \sigma$ level. Hence the precise observations of the Cosmic Microwave Background Radiation permit the testing of some fundamental predictions of inflation on primordial fluctuations, such as Gaussianity and scale independence [1-7].

More interestingly, the search for the background geometry and topology of the Universe reveals that a Bianchi pattern, corresponding to a homogeneous but anisotropic geometry of the Universe may account, in a quite efficient way, for some large-scale anomalies seen in the Planck data [5]. A Bayesian search for an anisotropic Bianchi $\mathrm{VII}_{\mathrm{h}}$ geometry was performed, by using the Planck data in [5]. In a non-physical setting, with the Bianchi parameters decoupled from the standard cosmology, the observational data favor a Bianchi component with a Bayes factor of at least 1.5 units of log-evidence. On the other hand, in the physically motivated setting where the Bianchi parameters are fitted simultaneously with the standard cosmological parameters, no indication for a Bianchi $\mathrm{VII}_{\mathrm{h}}$ cosmology was detected [5].

The statistical isotropy characterizing the large-scale structure of the Universe is an important prediction of cosmology, also supported by the cosmic no-hair conjecture according to which inflation eliminates classical (or even quantum) anisotropy. However, several recent observations of the large-scale structure of the Universe have questioned the principles of homogeneity and isotropy $[139,140]$. Some of the recent observations, not related to the study of the $\mathrm{CMB}$, and pointing towards possible anisotropies in the Universe are obtained from the investigations of Type Ia Super- novae, of the X-ray background, the distribution of the optical and infrared galaxies, and the observation of some peculiar velocities of the galaxy clusters [140] may raise some concerns about the absolute validity of the principle of isotropy. In [140] the directional behavior of the X-ray luminositytemperature relation $L_{X}-T$ of galaxy clusters was investigated. The measurement of the luminosity depends on the considered cosmological model via the luminosity distance $D_{L}$. On the other hand the temperature can be determined astrophysically without any cosmological assumptions. It was found that the behavior of the $L_{X}-T$ relation strongly depends on the direction of the sky, a result consistent with previous investigations. Strong anisotropies were detected at $a \geq 4 \sigma$ level. From the study of a sample of 142,661 quasars, with the data extending beyond the post-inflationary causality scales, significant spatially correlated systematic effects that can emulate cosmological anisotropy were found in [141]. When combined with the recent Planck results, these powerful observational results indicate that the presence of an intrinsic large-scale anisotropy in the Universe, of cosmological origin, cannot be ignored a priori.

The intriguing possibility that the geometry of the Universe may not be of the standard Friedmann-LemaitreRobertson-Walker (FLRW) form was investigated, from different points of view, in [142-153].

In particular, so-called extended FLRW models, representing a cosmological model with an underlying anisotropic Bianchi geometry that expands isotropically, and that can be mapped onto a standard FLRW model with the same expansion history, were investigated in [153]. It was found that matter and geometrical anisotropies tend to cancel out each other dynamically, and that, under rather general conditions, the expansion is asymptotically isotropic.

Hence, it turns out that there is a significant theoretical and empirical evidence for the existence of the large-scale cosmological anisotropies. However, the physical origin of anisotropy is still unknown, with the most favored explanations related to the deviations of the primordial fluctuations from isotropy [1]. However, up to now there no convincing physical mechanism that could lead to such deviations has been proposed.

A possible physical mechanism for the generation of the anisotropies in the Universe was proposed in [154], and it is based on the idea that the two major fluid components of the Universe, dark energy and dark matter, flow with distinct four-velocities. This cosmological configuration is equivalent to a single anisotropic fluid [155-158], expanding with a four-velocity representing a combination of the two fluid four-velocities. Therefore, if there is a slight difference between the four-velocities of the dark energy and dark matter, the Universe would acquire some anisotropic characteristics, and its geometry will deviate from the standard FLRW one, a conclusion that is supported by a number of present day 
observations. Along the same line of thought in [159] it was pointed out that there is no a priori reason to impose for the dark component of the Universe a reference frame comoving with ordinary matter. The consequences of relaxing this assumption were investigated through the study the cosmology of non-comoving fluids. Among the observable effects, observable modifications in the density-velocity and densitylensing potential cross-correlation spectra were found. The corrections from the non-comoving motion of the Universe components give rise to deviations from statistical isotropy with a dipolar structure. A two-fluid dark matter model, in which dark matter is represented as a two-component fluid thermodynamic system, without interaction between the constituent particles of different species, and with each distinct component having a different four-velocity, was considered in [160]. The properties of such a system were further investigated in [161], by assuming that the two dark matter components are pressureless, non-comoving fluids. For this particular choice of the equations of state the dark matter distribution can be described as a single anisotropic fluid, with vanishing tangential pressure, and non-zero radial pressure. The interesting possibility that there could exist different rest frames for dark matter and dark energy has been also studied in [162165]. The existence of large-scale bulk flows may represent some evidence for the presence of moving dark energy in the cosmological era when photons decoupled from matter.

It is the purpose of the present paper to extend the previous studies of the possibility of the non-comoving motion of the cosmological components to the very early Universe, and, more exactly, to the inflationary era. More specifically, we will consider the warm inflationary scenario, in which the early Universe is modeled as an interacting two-component fluid. In the standard warm inflationary scenario the assumption that all constituents of the early Universe move in the same rest frame, and with the same four-velocity, is a basic (but not exactly justifiable) hypothesis. However, it allows one to adopt a frame that is comoving with both the scalar field and the matter (radiation) constituents, thus allowing to chose all the components of the four-velocities $u^{\mu}$ as $u^{\mu}=(1,0,0,0)$. An important consequence of this assumption is that the global thermodynamic parameters of the inflationary Universe are just the sum of the individual thermodynamic parameters. Hence in the standard warm inflationary model the energy density of the early Universe $\rho$ is given by $\rho=\sum_{i=1}^{n} \rho_{i}$, where $\rho_{i}, i=1,2, \ldots, n$ are the energy densities of the individual constituents (generally scalar field and radiation, respectively).

However, there is no fundamental observational or physical principle that would require that all matter and energy constituents in the early Universe must have the same fourvelocity. Hence there is no a priori reason to describe the dynamics of the inflationary Universe in a single frame, comoving with the scalar field as well as the matter con- stituents. In the following we will investigate the warm inflationary model by assuming that the very early Universe can be described as a mixture of two interacting perfect fluids, namely scalar field and radiation, respectively, possessing different four-velocities. Therefore the two components non-comoving early Universe becomes formally equivalent to a single anisotropic fluid, as already pointed out in [154-158,160,161]. Therefore, if there is a slight difference between the four-velocities of the scalar field and radiation, the very early Universe would achieve some anisotropic characteristics, and its geometry and its expansionary evolution will not be anymore the standard FLRW one.

In our present study we assume that the scalar field and the radiation have distinct four-velocities. By using a rotation on the velocity space we can transform the energy-momentum tensor of the non-comoving two-fluid system to the standard form of anisotropic fluids. Due to this procedure the thermodynamic parameters (energy densities and pressures of the scalar field and of the radiation) of the warmly inflating Universe are represented in terms of a single fluid, described by an anisotropic energy-momentum tensor. The energy density of the single cosmological fluid is greater than the sum of the energy densities of the scalar field and of the radiation, respectively, and it contains a supplementary term due to the anisotropy induced by the non-comoving motion. For the very early Universe we assume the simplest case of a Bianchi Type I geometry, which is a consequence of the noncomoving expansion of the scalar field and radiation fluid, respectively. For this system we obtain the anisotropic gravitational field equations and the generalized Klein-Gordon equation for the scalar field.

Once the general formalism is developed, we implement the idea of the warm inflation by introducing the decay terms for the scalar field and the radiation. Such a splitting of the energy conservation equation for the scalar field and radiation gives the decay equations for the scalar field, and for the radiation creation. As opposed to the standard warm inflationary scenario, the source terms for scalar field decay and radiation creation are not equal, with the radiation balance equation containing an anisotropic term proportional to the Hubble factor along the $z$ direction. We investigate in detail the slowroll approximation of the model, as well as its consistency with observations, in the two standard limits usually considered in the literature, the weak dissipation and the strong dissipation limits, respectively. Then the theoretical predictions of the warm inflationary scenario with non-comoving scalar field and radiation fluid are compared in detail with the observational data obtained by the Planck satellite, and constraints on the free parameters of the model are obtained. The functional forms of the scalar field potentials, compatible with the non-comoving nature of warm inflation, are also obtained by considering some distinct choices for the scale factors. 
The present paper is organized as follows. The reformulation of the energy-momentum tensor of the scalar fieldradiation fluid two-component system to a single effective anisotropic fluid energy-momentum tensor is presented in Sect. 2. The warm inflationary model with non-comoving scalar field and radiation is introduced in Sect. 3, where the balance equations for the scalar field and radiation are obtained for a Bianchi Type I geometry. The slow-roll approximation is considered in Sect. 4. We discuss and conclude our results in Sect. 5.

\section{Anisotropic fluid description of the non-comoving scalar-field-radiation cosmological models}

We assume that the early Universe consisted of a mixture of two basic fluid components: a scalar field $\phi$, with energy density and pressure $\rho_{\phi}$ and $p_{\phi}$, and four-velocity $u_{\phi}^{\mu}$, respectively, and radiation, characterized by the thermodynamical parameters $\rho_{\text {rad }}$ and $p_{\text {rad }}$, respectively, and four-velocity $u_{\text {rad }}^{\mu}$. The dynamical evolution of the system can then be obtained from the variational principle,

$S=\int\left[\frac{M_{P l}^{2}}{16 \pi} R+\frac{1}{2} \nabla_{\mu} \phi \nabla^{\mu} \phi-U(\phi)+L_{\mathrm{rad}}\right] \sqrt{-g} \mathrm{~d}^{4} x$,

where $M_{P l}=G^{-1 / 2}$ is the Planck mass, $U(\phi)$ is the selfinteraction potential of the scalar field, and $L_{\text {rad }}$ is the radiation Lagrangian. The Lagrangian density of the scalar field is $L_{\phi}=(1 / 2) \nabla_{\mu} \phi \nabla^{\mu} \phi-U(\phi)$. By $\nabla_{\mu}$ we denote the covariant derivative with respect to the metric.

\subsection{Four-velocities and energy-momentum tensors}

Now by varying the total action with respect to the metric and the scalar field $\phi$, we obtain the energy-momentum tensor of the system as $T^{\mu \nu}=T_{(\phi)}^{\mu \nu}+T_{(\mathrm{rad})}^{\mu \nu}$, where $T_{(\phi)}^{\mu \nu}=\nabla^{\mu} \phi \nabla^{v} \phi-$ $L_{\phi} g^{\mu \nu}$.

We would like now to reformulate the energy-momentum of the scalar field in a form similar to that of the energymomentum tensor of a perfect fluid. For this we need to introduce a quantity $u_{(\phi)}^{\mu}$, having properties similar to the four-velocity of the perfect fluid, that is, satisfying the conditions $g_{\mu \nu} u_{(\phi)}^{\mu} u_{(\phi)}^{v}=1$, and the vector is timelike. Such a vector can be constructed as $[166,167]$

$u_{(\phi)}^{\mu}=\frac{\nabla^{\mu} \phi}{\sqrt{\nabla_{\nu} \phi \nabla^{\nu} \phi}}$.

For such a construction to be physically acceptable, that is, for $u_{(\phi)}^{\mu}$ to be real and timelike, the scalar field must satisfy the conditions that $\nabla^{\mu} \phi$ is real, and $\nabla_{\nu} \phi \nabla^{\nu} \phi>0$. Then we can associate to the scalar field an effective energy density $\rho_{\phi}$ and pressure $p_{\phi}$, given by $\rho_{\phi}=T_{(\phi)}^{\mu v} u_{(\phi) \mu} u_{(\phi) v}$, and $p_{\phi}=(1 / 3) \Pi_{\mu}^{\mu}$, where $\Pi_{\mu \nu}=-T_{(\phi) \sigma \rho} h_{\mu}^{\sigma} h_{\nu}^{\rho}$, where $h_{\mu}^{\sigma}=u_{(\phi)}^{\sigma} u_{(\phi) \mu}-\delta_{\mu}^{\sigma}$ is the projection operator. Then, by using these definitions, one can easily obtain

$\rho_{\phi}=\frac{1}{2} \nabla_{\mu} \phi \nabla^{\mu} \phi+U(\phi), \quad p_{\phi}=\frac{1}{2} \nabla_{\mu} \phi \nabla^{\mu} \phi-U(\phi)$

The energy-momentum tensor of the scalar field can then be written in a form similar to the perfect fluid case,

$T_{(\phi)}^{\mu \nu}=\left(\rho_{\phi}+p_{\phi}\right) u_{(\phi)}^{\mu} u_{(\phi)}^{\nu}-p_{\phi} g^{\mu \nu}$.

The thermodynamical and physical properties of the mixture of scalar field and radiation cosmological fluid can be obtained from the total energy-momentum tensor $T^{\mu \nu}$ of the Universe, given by the sum of each individual components as

$$
\begin{aligned}
T^{\mu \nu}= & T_{(\phi)}^{\mu \nu}+T_{(\mathrm{rad})}^{\mu \nu}=\left(\rho_{\phi}+p_{\phi}\right) u_{(\phi)}^{\mu} u_{(\phi)}^{\nu}-p_{\phi} g^{\mu \nu} \\
& +\left(\rho_{\mathrm{rad}}+p_{\mathrm{rad}}\right) u_{(\mathrm{rad})}^{\mu} u_{(\mathrm{rad})}^{\nu}-p_{\mathrm{rad}} g^{\mu \nu} .
\end{aligned}
$$

The four-velocities of the scalar field and of the radiation fluid are normalized according to $u_{(\phi)}^{\mu} u_{(\phi) \mu}=1$ and $u_{(\mathrm{rad})}^{\mu} u_{(\mathrm{rad})} \mu=1$, respectively. As for the radiation fluid we will adopt the standard equation of state for the photon gas, $p_{\text {rad }}=(1 / 3) \rho_{\text {rad }}$.

The total energy-momentum of the mixture of the scalar field and radiation must satisfy the conservation equation $\nabla_{\mu} T^{\mu \nu}=0$, which gives

$$
\begin{aligned}
\nabla_{\mu} T^{\mu v}= & \left(\nabla_{\mu} \rho_{\phi}+\nabla_{\mu} p_{\phi}\right) u_{(\phi)}^{\mu} u_{(\phi)}^{v} \\
& +\left(\rho_{\phi}+p_{\phi}\right) \nabla_{\mu} u_{(\phi)}^{\mu} u_{(\phi)}^{v} \\
& +\left(\rho_{\phi}+p_{\phi}\right) u_{(\phi)}^{\mu} \nabla_{\mu} u_{(\phi)}^{v} \\
& -\nabla_{\mu} p_{\phi} g^{\mu v}+\nabla_{\mu} T_{(\mathrm{rad})}^{\mu \nu}=0 .
\end{aligned}
$$

After multiplication of the above equation by $u_{(\phi) v}$, and by taking into account the mathematical identity $u_{(\phi) \nu} \nabla_{\mu} u^{(\phi) v}=$ 0 , the energy conservation equation of the mixture of two fluids takes the form

$\dot{\rho}_{\phi}+3 H_{\phi}\left(\rho_{\phi}+p_{\phi}\right)+u_{(\phi) \nu} \nabla_{\mu} T_{(\mathrm{rad})}^{\mu \nu}=0$,

where we have denoted $(. .)=.u_{(\phi) \nu} \nabla^{\mu}(\ldots)$, while $H_{\phi}=$ $(1 / 3) \nabla_{\mu} u^{(\phi) \mu}$. Alternatively, by using the radiation fluid quantities the energy conservation equation can be formulated as

$\dot{\rho}_{\text {rad }}+3 H_{\text {rad }}\left(\rho_{\text {rad }}+p_{\text {rad }}\right)+u_{(\text {rad }) \nu} \nabla_{\mu} T_{(\phi)}^{\mu \nu}=0$,

where in the case of radiation $(. .)=.u_{(\mathrm{rad}) \nu} \nabla^{\mu}(\ldots)$, while $H_{\mathrm{rad}}=(1 / 3) \nabla_{\mu} u^{(\mathrm{rad}) \mu}$. Equations (7) and (8) give the evolution of the energy densities of the scalar field and radiation 
fluid, indicating the possibility of the energy transfer from one component to the other. In order for the two fluids to expand at the same rate the condition $H_{\phi}=H_{\text {rad }}$ must be satisfied, giving the condition $\nabla_{\mu} u^{(\phi) \mu}=\nabla_{\mu} u^{(\mathrm{rad}) \mu}$. This equation has the obvious solution $u^{(\phi) \mu}=u^{(\mathrm{rad}) \mu}$. But if we interpret it mathematically as a partial differential equation for either $u^{(\phi) \mu}$ or $u^{(\phi) \mu}$, then more general solutions with $u^{(\phi) \mu}=u^{(\phi) \mu}\left(u^{(\mathrm{rad}) v}, x^{\lambda}\right)$ are also possible, and their existence is ensured by the condition of the existence and unicity of the solutions of first order partial differential equations.

In standard cosmology it is usually assumed that the two cosmological fluids are comoving, which implies

$u_{(\phi)}^{\mu} \equiv u_{(\mathrm{rad})}^{\mu} \equiv u^{\mu}, \quad \mu=0,1,2,3$.

This condition allows one to choose for the study of the cosmological dynamics a comoving frame, in which all the components of the four velocity of all cosmic constituents can be reformulated as $u^{\mu}=(1,0,0,0)$. In the case when $u_{(\phi)}^{\mu}=u_{(\mathrm{rad})}^{\mu} \equiv w^{\mu}$, the energy-momentum tensor of the scalar field plus radiation system takes the simple form

$T^{\mu \nu}=\left(\rho_{\phi}+p_{\phi}+\rho_{\mathrm{rad}}+p_{\mathrm{rad}}\right) w^{\mu} w^{\nu}-\left(p_{\phi}+p_{\mathrm{rad}}\right) g^{\mu \nu}$.

In this case the scalar field-radiation fluid system reduces to an isotropic effective single fluid system. Hence, if the scalar field and the photon gas have the same four-velocity, the thermodynamic parameters of the scalar field-radiation two-fluid system are obtained a simple addition of the enthalpies and the pressures of the individual components. For such a physical system one can always introduce a comoving frame, in which the components of the fourvelocity are $w^{\mu}=(1,0,0,0)$, with the components of the total energy-momentum tensor of the two fluids given by $T_{0}^{0}=\left(\rho_{\phi}+\rho_{\mathrm{rad}}\right) \delta_{0}^{0}$, and $T_{i}^{i}=-\left(p_{\phi}+p_{\mathrm{rad}}\right) \delta_{i}^{i}, i=$ $1,2,3$, no summation over $i$.

However, in the present study of warm inflation we will abandon this condition, by assuming that, during at least some intervals of the cosmological expansion, the scalar field and the radiation components of the warm inflationary Universe may have had different four-velocities, so that

$u_{(\phi)}^{\mu} \neq u_{(\mathrm{rad})}^{\mu}, \quad \mu=0,1,2,3$.

In such a situation it is not possible to introduce a comoving frame, so that the thermodynamical quantities are constructed as the sum of the thermodynamical parameters of each component of the mixture. In our analysis of the warm inflationary scenarios in non-comoving frames we will assume that the energy density and the pressure of the scalar field satisfy the condition $\rho_{\phi}+p_{\phi} \geq 0$, that is, the scalar field cannot be interpreted as a simple cosmological constant.
2.2 Single anisotropic fluid representation of two-fluid systems

The investigation of warm inflationary cosmological models in which the total matter content is described by an energy-momentum tensor having the representation given by Eq. (5) can be essentially simplified if we transform it into the standard form of the energy-momentum tensor of perfect anisotropic fluids. This can be achieved by means of the four-velocity transformations

$u_{(\phi)}^{\mu} \rightarrow u_{(\phi)}^{* \mu}, \quad u_{(\mathrm{rad})}^{\mu} \rightarrow u_{(\mathrm{rad})}^{* \mu}$,

respectively, with the transformation matrix given by [155158]

$$
\begin{aligned}
\left(\begin{array}{c}
u_{(\phi)}^{* \mu} \\
u_{(\mathrm{rad})}^{* \mu}
\end{array}\right)= & \left(\begin{array}{cc}
\cos \alpha & \sqrt{\frac{\rho_{\mathrm{rad}}+p_{\mathrm{rad}}}{\rho_{\phi}+p_{\phi}}} \sin \alpha \\
-\sqrt{\frac{\rho_{\phi}+p_{\phi}}{\rho_{\mathrm{rad}}+p_{\mathrm{rad}}}} \sin \alpha & \cos \alpha
\end{array}\right) \\
& \times\left(\begin{array}{c}
u_{(\phi)}^{\mu} \\
u_{(\mathrm{rad})}^{\mu}
\end{array}\right),
\end{aligned}
$$

or, equivalently,

$$
\begin{aligned}
\left(\begin{array}{c}
u_{(\phi)}^{* \mu} \\
u_{(\mathrm{rad})}^{* \mu}
\end{array}\right)= & \left(\begin{array}{cc}
\cos \alpha & \sqrt{\frac{4}{3} \frac{\rho_{\mathrm{rad}}}{\nabla_{\mu} \phi \nabla^{\mu} \phi}} \sin \alpha \\
-\sqrt{\frac{3}{4} \frac{\nabla_{\mu} \phi \nabla^{\mu} \phi}{\rho_{\mathrm{rad}}}} \sin \alpha & \cos \alpha
\end{array}\right) \\
& \times\left(\begin{array}{c}
u_{(\phi)}^{\mu} \\
u_{(\mathrm{rad})}^{\mu}
\end{array}\right) .
\end{aligned}
$$

This transformation represents a rotation of the fourvector velocities in the $\left(u_{(\phi)}^{\mu}, u_{(\mathrm{rad})}^{\mu}\right)$ velocity space. Explicitly, the transformations (14) take the form

$$
\begin{aligned}
u_{(\phi)}^{\mu} \rightarrow u_{(\phi)}^{* \mu}=u_{(\phi)}^{\mu} \cos \alpha+\sqrt{\frac{4}{3} \frac{\rho_{\mathrm{rad}}}{\nabla_{\mu} \phi \nabla^{\mu} \phi}} & u_{\mathrm{rad})}^{\mu} \sin \alpha, \\
u_{(\mathrm{rad})}^{\mu} \rightarrow u_{(\mathrm{rad})}^{* \mu}= & -\sqrt{\frac{3}{4} \frac{\nabla_{\mu} \phi \nabla^{\mu} \phi}{\rho_{(\mathrm{rad})}}} u_{(\phi)}^{\mu} \sin \alpha \\
& +u_{(\mathrm{rad})}^{\mu} \cos \alpha
\end{aligned}
$$

respectively.

The transformations in the velocity space given by Eq. (13) leave the quadratic form $\left(\rho_{\phi}+p_{\phi}\right) u_{(\phi)}^{\mu} u_{(\phi)}^{\nu}+$ $\left(\rho_{\mathrm{rad}}+p_{\mathrm{rad}}\right) u_{(\mathrm{rad})}^{\mu} u_{(\mathrm{rad})}^{v}$ invariant. Thus,

$T^{\mu \nu}\left(u_{(\phi)}^{\mu}, u_{(\mathrm{rad})}^{\nu}\right)=T^{\mu \nu}\left(u_{(\phi)}^{* \mu}, u_{(\mathrm{rad})}^{* \nu}\right)$.

As a next step in our analysis we choose $u_{(\phi)}^{* \mu}$ and $u_{(\mathrm{rad})}^{* \mu}$ so that one becomes timelike, while the other one is spacelike. Moreover, we also assume that the two new four-vector velocities satisfy the orthogonality condition

$u_{(\phi)}^{* \mu} u_{(\mathrm{rad}) \mu}^{*}=0$. 
By using Eqs. (15) and (16) and the orthogonality condition as given by Eq. (18), we obtain for the rotation angle the expression

$$
\begin{aligned}
\tan 2 \alpha & =2 \frac{\sqrt{\left(\rho_{\phi}+p_{\phi}\right)\left(\rho_{\mathrm{rad}}+p_{\mathrm{rad}}\right)}}{\rho_{\phi}+p_{\phi}-\rho_{\mathrm{rad}}-p_{\mathrm{rad}}} u_{(\phi)}^{\mu} u_{(\mathrm{rad}) \mu} \\
& =2 \frac{\sqrt{4\left(\nabla_{\mu} \phi \nabla^{\mu} \phi\right) \rho_{\mathrm{rad}} / 3}}{\nabla_{\mu} \phi \nabla^{\mu} \phi-4 \rho_{\mathrm{rad}} / 3} u_{(\phi)}^{\mu} u_{(\mathrm{rad}) \mu} .
\end{aligned}
$$

If the angle $\alpha$ has a different form, and it is not given by Eq. (19), we cannot find a transformed spacelike $u_{(\phi)}^{* \mu}$, and a timelike $u_{(\mathrm{rad})}^{* \mu}$ scalar field and radiation fluid velocity, respectively. Note that, if $\rho_{\phi}+p_{\phi}=0$, a case which corresponds to the cosmological constant, the rotation angle is $\alpha=0$. Consequently, the rotation in the velocity space reduces to the identical transformation. Hence in the presence of a cosmological constant, since $\rho_{\phi}+p_{\phi}=0$, the term $\left(\rho_{\phi}+p_{\phi}\right) u_{(\phi)}^{\mu} u_{(\phi)}^{\nu}$ in the energy-momentum tensor of the scalar field is identically equal to zero, $\left(\rho_{\phi}+p_{\phi}\right) u_{(\phi)}^{\mu} u_{(\phi)}^{v} \equiv$ 0 . Therefore the four-velocity of the cosmological constant does not appear in the above introduced formalism. Hence the effective energy-momentum tensor of cosmological system consisting of a pure cosmological constant plus radiation fluid can be equivalently described by the energy-momentum tensor of a single isotropic fluid.

Next, we introduce the new set of quantities $\left(V^{\mu}\right.$, $\left.\chi^{\mu}, \varepsilon, \Psi, \Pi\right)$ defined according to

$$
\begin{aligned}
& V^{\mu}=\frac{u_{(\phi)}^{* \mu}}{\sqrt{u_{(\phi)}^{* \alpha} u_{(\phi) \alpha}^{*}}}, \chi^{\mu}=\frac{u_{(\mathrm{rad})}^{* \mu}}{\sqrt{-u_{(\mathrm{rad})}^{* \alpha} u_{(\mathrm{rad}) \alpha}^{*}}} \\
& \varepsilon=T^{\mu v} V_{\mu} V_{v}=\left(\rho_{\phi}+p_{\phi}\right) u_{(\phi)}^{* \alpha} u_{(\phi) \alpha}^{*}-\left(p_{\phi}+p_{\mathrm{rad}}\right) \\
& \Psi=T^{\mu v} \chi_{\mu} \chi_{v}=\left(p_{\phi}+p_{\mathrm{rad}}\right) \\
& -\left(\rho_{\mathrm{rad}}+p_{\mathrm{rad}}\right) u_{(\mathrm{rad})}^{* \alpha} u_{(\mathrm{rad}) \alpha}^{*}
\end{aligned}
$$

and

$\Pi=p_{\phi}+p_{\mathrm{rad}}$

respectively, where from a physical point of view $\varepsilon$ can be interpreted as the energy density and $\Psi$ as the radial pressure of an anisotropic Universe. Then, by adopting this interpretation, it turns out that the energy-momentum tensor of the non-comoving scalar field and radiation fluids can be written as

$T^{\mu \nu}=(\varepsilon+\Pi) V^{\mu} V^{v}-\Pi g^{\mu \nu}+(\Psi-\Pi) \chi^{\mu} \chi^{v}$,

where $V^{\mu} V_{\mu}=1=-\chi^{\mu} \chi_{\mu}$ and $\chi^{\mu} V_{\mu}=0$ [155-158].

The energy-momentum tensor of the two-fluid warm inflationary cosmological model, in which the components have different four-velocities, given by Eq. (24), is the standard form of the energy-momentum tensor for anisotropic fluids [158].

The energy density $\varepsilon$ and the radial pressure $\Psi$ of the scalar field and radiation fluid filled Universe are given by

$$
\begin{aligned}
\varepsilon= & \frac{1}{2}\left(\rho_{\phi}-p_{\phi}+\rho_{\mathrm{rad}}-p_{\mathrm{rad}}\right)+\frac{1}{2} \\
& \times\left\{\left(\rho_{\phi}+p_{\phi}+\rho_{\mathrm{rad}}+p_{\mathrm{rad}}\right)^{2}\right. \\
& +4\left(\rho_{\phi}+p_{\phi}\right)\left(\rho_{\mathrm{rad}}+p_{\mathrm{rad}}\right) \\
& \left.\times\left[\left(u_{(\phi)}^{\mu} u_{(\mathrm{rad}) \mu}\right)^{2}-1\right]\right\}^{1 / 2}
\end{aligned}
$$

and

$$
\begin{aligned}
\Psi= & -\frac{1}{2}\left(\rho_{\phi}-p_{\phi}+\rho_{\mathrm{rad}}-p_{\mathrm{rad}}\right)+\frac{1}{2} \\
& \times\left\{\left(\rho_{\phi}+p_{\phi}+\rho_{\mathrm{rad}}+p_{\mathrm{rad}}\right)^{2}\right. \\
& +4\left(\rho_{\phi}+p_{\phi}\right)\left(\rho_{\mathrm{rad}}+p_{\mathrm{rad}}\right) \\
& \left.\times\left[\left(u_{(\phi)}^{\mu} u_{(\mathrm{rad}) \mu}\right)^{2}-1\right]\right\}^{1 / 2},
\end{aligned}
$$

respectively [155-158]. Explicitly, the energy density and the radial pressure of the non-comoving scalar field-radiation fluid system can be obtained:

$$
\begin{aligned}
\varepsilon= & U(\phi)+\frac{\rho_{\mathrm{rad}}}{3}+\frac{1}{2}\left\{\left(\nabla_{\mu} \phi \nabla^{\mu} \phi+\frac{4}{3} \rho_{\mathrm{rad}}\right)^{2}\right. \\
+ & \left.\frac{16}{3} \rho_{\mathrm{rad}} \nabla_{\mu} \phi \nabla^{\mu} \phi\left[\left(u_{(\phi)}^{\mu} u_{(\mathrm{rad}) \mu}\right)^{2}-1\right]\right\}^{1 / 2}, \\
\Psi= & -U(\phi)-\frac{\rho_{\mathrm{rad}}}{3}+\frac{1}{2}\left\{\left(\nabla_{\mu} \phi \nabla^{\mu} \phi+\frac{4}{3} \rho_{\mathrm{rad}}\right)^{2}\right. \\
& \left.+\frac{16}{3} \rho_{\mathrm{rad}} \nabla_{\mu} \phi \nabla^{\mu} \phi\left[\left(u_{(\phi)}^{\mu} u_{(\mathrm{rad}) \mu}\right)^{2}-1\right]\right\}^{1 / 2} .
\end{aligned}
$$

The energy density of the anisotropic effective fluid, describing the scalar field and radiation non-comoving mixture, given by Eq. (25), depends explicitly on the expressions of the four-velocities of the two fluids. Therefore, the energy density of the rotated fluids also depends on the kinetic energy of the scalar field and of the radiation fluid, and this functional dependence implies that the energy in the rotated fourvelocity space is different from the sum of the rest mass energies of the scalar field and of radiation, respectively. When $u_{(\phi)}^{\mu}=u_{(\mathrm{rad})}^{\mu}$, then $u_{(\phi)}^{\mu} u_{(\mathrm{rad}) \mu}=1$, and the corresponding expressions of the effective energy and pressure of the fluid reduce to the sum of the energy densities of the scalar field and of the radiation fluid, respectively, so that $\varepsilon=\rho_{\phi}+\rho_{\text {rad }}$, $\Psi=p_{\phi}+p_{\text {rad }}$. Moreover, in this case one can adopt a comoving frame to describe the cosmological dynamics. 
Since the two cosmological fluids, the scalar field and radiation, have different four-velocities, we can write

$u_{(\phi)}^{\mu} u_{(\mathrm{rad}) \mu}=1+\frac{b}{2}$,

where in the general case $b$ is an arbitrary function of the energy densities and pressures of the scalar field and of the radiation, respectively. The exact expression of $b=$ $b\left(\rho_{\phi}, p_{\phi}, \rho_{\text {rad }}, p_{\text {rad }}\right)$ can be obtained from Eq. (19), which gives first

$u_{(\phi)}^{\mu} u_{(\mathrm{rad}) \mu}=\frac{1}{2}\left[\sqrt{\frac{\rho_{\phi}+p_{\phi}}{\rho_{\mathrm{rad}}+p_{\mathrm{rad}}}}-\sqrt{\frac{\rho_{\mathrm{rad}}+p_{\mathrm{rad}}}{\rho_{\phi}+p_{\phi}}}\right] \tan 2 \alpha$.

Then the functional form of $b$ can be determined immediately as

$b=\left[\sqrt{\frac{\rho_{\phi}+p_{\phi}}{\rho_{\mathrm{rad}}+p_{\mathrm{rad}}}}-\sqrt{\frac{\rho_{\mathrm{rad}}+p_{\mathrm{rad}}}{\rho_{\phi}+p_{\phi}}}\right] \tan 2 \alpha-2$.

In terms of $b$, Eqs. (25) and (26) can be reformulated as

$$
\begin{aligned}
\varepsilon= & \frac{1}{2}\left(\rho_{\phi}-p_{\phi}+\rho_{\mathrm{rad}}-p_{\mathrm{rad}}\right) \\
& +\frac{1}{2}\left(\rho_{\phi}+p_{\phi}+\rho_{\mathrm{rad}}+p_{\mathrm{rad}}\right) \\
& \times\left[1+4 b\left(1+\frac{b}{4}\right) \frac{\left(\rho_{\phi}+p_{\phi}\right)\left(\rho_{\mathrm{rad}}+p_{\mathrm{rad}}\right)}{\left(\rho_{\phi}+p_{\phi}+\rho_{\mathrm{rad}}+p_{\mathrm{rad}}\right)^{2}}\right]^{1 / 2}
\end{aligned}
$$

and

$$
\begin{aligned}
\Psi= & -\frac{1}{2}\left(\rho_{\phi}-p_{\phi}+\rho_{\mathrm{rad}}-p_{\mathrm{rad}}\right) \\
& +\frac{1}{2}\left(\rho_{\phi}+p_{\phi}+\rho_{\mathrm{rad}}+p_{\mathrm{rad}}\right) \\
& \times\left[1+4 b\left(1+\frac{b}{4}\right) \frac{\left(\rho_{\phi}+p_{\phi}\right)\left(\rho_{\mathrm{rad}}+p_{\mathrm{rad}}\right)}{\left(\rho_{\phi}+p_{\phi}+\rho_{\mathrm{rad}}+p_{\mathrm{rad}}\right)^{2}}\right]^{1 / 2}
\end{aligned}
$$

Explicitly, we obtain

$$
\begin{aligned}
\varepsilon= & U(\phi)+\frac{\rho_{\mathrm{rad}}}{3}+\frac{1}{2}\left(\nabla_{\mu} \phi \nabla^{\mu} \phi+\frac{4}{3} \rho_{\mathrm{rad}}\right) \\
& \times \sqrt{1+\frac{16 b}{3}\left(1+\frac{b}{4}\right) \frac{\nabla_{\mu} \phi \nabla^{\mu} \phi \rho_{\mathrm{rad}}}{\left(\nabla_{\mu} \phi \nabla^{\mu} \phi+\frac{4}{3} \rho_{\mathrm{rad}}\right)^{2}}}
\end{aligned}
$$

and

$$
\begin{aligned}
\Psi= & -U(\phi)-\frac{\rho_{\mathrm{rad}}}{3}+\frac{1}{2}\left(\nabla_{\mu} \phi \nabla^{\mu} \phi+\frac{4}{3} \rho_{\mathrm{rad}}\right) \\
& \times \sqrt{1+\frac{16 b}{3}\left(1+\frac{b}{4}\right) \frac{\nabla_{\mu} \phi \nabla^{\mu} \phi \rho_{\mathrm{rad}}}{\left(\nabla_{\mu} \phi \nabla^{\mu} \phi+\frac{4}{3} \rho_{\mathrm{rad}}\right)^{2}}} .
\end{aligned}
$$

By assuming that the physical parameters of the scalar field and of the radiation fluid satisfy the condition

$4 b\left(1+\frac{b}{4}\right) \frac{\left(\rho_{\phi}+p_{\phi}\right)\left(\rho_{\mathrm{rad}}+p_{\mathrm{rad}}\right)}{\left(\rho_{\phi}+p_{\phi}+\rho_{\mathrm{rad}}+p_{\mathrm{rad}}\right)^{2}} \ll 1$,

or

$b\left(1+\frac{b}{4}\right) \frac{\nabla_{\mu} \phi \nabla^{\mu} \phi \rho_{\mathrm{rad}}}{\left(\nabla_{\mu} \phi \nabla^{\mu} \phi+\frac{4}{3} \rho_{\mathrm{rad}}\right)^{2}} \ll \frac{3}{16}$,

after series expanding the square root in Eqs. (32) and (26), and keeping only the first order of approximation, it follows that the energy density, the radial and the tangential pressures of the Universe filled with a scalar field and a radiation fluid can be obtained:

$$
\begin{aligned}
\varepsilon & =\rho_{\phi}+\rho_{\mathrm{rad}}+F\left(\rho_{\phi}, \rho_{\mathrm{rad}}\right) \\
& =\frac{1}{2} \nabla_{\mu} \phi \nabla^{\mu} \phi+U(\phi)+\rho_{\mathrm{rad}}+F\left(\rho_{\phi}, \rho_{\mathrm{rad}}\right), \\
\Psi & =p_{\phi}+p_{\mathrm{rad}}+F\left(\rho_{\phi}, \rho_{\mathrm{rad}}\right) \\
& =\frac{1}{2} \nabla_{\mu} \phi \nabla^{\mu} \phi-U(\phi)+p_{\mathrm{rad}}+F\left(\rho_{\phi}, \rho_{\mathrm{rad}}\right), \\
\Pi & =p_{\phi}+p_{\mathrm{rad}}
\end{aligned}
$$

where we have denoted

$$
\begin{aligned}
F\left(\rho_{\phi}, \rho_{\mathrm{rad}}\right)= & 2 b\left(1+\frac{b}{4}\right) \frac{\left(\rho_{\phi}+p_{\phi}\right)\left(\rho_{\mathrm{rad}}+p_{\mathrm{rad}}\right)}{\left(\rho_{\phi}+p_{\phi}+\rho_{\mathrm{rad}}+p_{\mathrm{rad}}\right)} \\
& =\frac{8 b}{3}\left(1+\frac{b}{4}\right) \frac{\nabla_{\mu} \phi \nabla^{\mu} \phi \rho_{\mathrm{rad}}}{\left(\nabla_{\mu} \phi \nabla^{\mu} \phi+4 \rho_{\mathrm{rad}} / 3\right)}
\end{aligned}
$$

By assuming that $\rho_{\phi}+p_{\phi}>>\rho_{\text {rad }}+p_{\text {rad }}$, we obtain for $F$ the expression

$F\left(\rho_{\phi}, \rho_{\mathrm{rad}}\right) \approx \frac{8 b}{3}\left(1+\frac{b}{4}\right) \rho_{\mathrm{rad}}$.

In the limit $b / 4>>1$, we obtain

$F\left(\rho_{\phi}, \rho_{\mathrm{rad}}\right) \approx \frac{2}{3} b^{2} \rho_{\mathrm{rad}}$, 
while in the opposite limit, $b / 4<<1, F\left(\rho_{\phi}, \rho_{\text {rad }}\right)$ can be approximated by

$F\left(\rho_{\phi}, \rho_{\text {rad }}\right) \approx \frac{8 b}{3} \rho_{\text {rad }}$.

As one can easily see from Eq. (38), the total energy density of the non-comoving scalar field and radiation fluid filled Universe is different from the sum of the energy densities of the two fluids. Moreover, a coupling between the energy densities of the scalar field and of the radiation is naturally generated in this scenario.

\section{Warm inflation with non-comoving scalar field and radiation}

In the present section, we consider the cosmological applications of the non-comoving scalar field-radiation fluid physical system. More exactly, we will investigate this model in the framework of the warm inflationary scenario, in which it is assumed that the very early Universe consisted of a scalar field that decayed into a radiation fluid. We will assume that the four-velocity of the scalar field was not exactly equal with the four-velocity of the photon gas. Therefore, in such a situation, one cannot introduce a comoving frame to describe the global evolution. Moreover, the non-comoving two-fluid system becomes anisotropic, and the total energy and pressure of the system contains an interaction term between the energy densities and pressures of the scalar field and the radiation fluid.

As a first step in our analysis of this warm inflationario scenario, we write down the gravitational field equations corresponding to the anisotropic evolution of the Universe filled with interacting scalar field and radiation fluid. We analyze then the general properties of the model, and we show that the non-comoving nature of the cosmological dynamics induces some specific anisotropic effects in the evolution of both expansion and shear parameters. We also consider specific non-comoving warm inflationary models, and the general solution of the field equations, describing the scalar field-radiation fluid mixture is obtained.

\subsection{Brief review of the warm inflationary scenario}

The warm inflationary model $[81,82]$ is an interesting and elegant theoretical alternative to the cold inflation and reheating scenarios. Similarly to standard inflationary theories, in warm inflation the Universe also experiences an accelerated, de Sitter type, very early expansionary stage, which is triggered by the presence of the scalar field, representing the dominant cosmological component. But, as opposed to the cold inflation scenario, besides a scalar field, a matter component of the cosmological fluid, usually assumed to exist in the form of radiation, is also present. The matter component is generated by the scalar field all along the accelerating expansion phase. During the cosmological evolution, these two components interact dynamically. The cosmological evolution is still described by the standard Friedmann equations,

$$
\begin{aligned}
& 3 H^{2}=\frac{1}{M_{P}^{2}}\left(\rho_{\phi}+\rho_{\mathrm{rad}}\right), \\
& 2 \dot{H}=-\frac{1}{M_{P}^{2}}\left(\dot{\phi}^{2}+\frac{4}{3} \rho_{\mathrm{rad}}\right),
\end{aligned}
$$

where by $M_{P}$ we have denoted the Planck mass. The energy density and the pressure of the scalar field are given, as usual, by $\rho_{\phi}=\dot{\phi}^{2} / 2+U(\phi)$ and $p_{\phi}=\dot{\phi}^{2} / 2-U(\phi)$, respectively, where $U(\phi)$ is the scalar field self-interaction potential. Due to the decay of the scalar field, which is essentially a dissipative process, energy is transferred from the field to radiation, and this process is described by the following energy balance equations:

$$
\begin{aligned}
& \dot{\rho}_{\phi}+3 H\left(\rho_{\phi}+p_{\phi}\right)=-\Gamma \dot{\phi}^{2}, \\
& \dot{\rho}_{\mathrm{rad}}+3 H\left(\rho_{\mathrm{rad}}+p_{\mathrm{rad}}\right)=\Gamma \dot{\phi}^{2},
\end{aligned}
$$

where $\Gamma$ is the dissipation coefficient. By using the explicit expressions of the energy density and pressure of the scalar field, Eq. (47) can be reformulated as the generalized KleinGordon equation for the scalar field,

$\ddot{\phi}+3 H(1+Q) \dot{\phi}+U^{\prime}(\phi)=0$,

where we have denoted $Q=\Gamma / 3 H$. By assuming that the cosmological expansion is quasi-de Sitter, that the scalar field energy density is much bigger than the energy density of the radiation, $\rho_{\phi}>>\rho_{\text {rad }}$, and that the potential term of the scalar field energy density dominates the kinetic one, so that $\rho_{\phi} \approx U(\phi)$, Eqs. (45), (48), and (49) can be approximated by

$$
\begin{aligned}
& 3 H^{2} \approx \frac{1}{M_{P}^{2}} U(\phi), \quad \dot{\phi} \approx-\frac{U^{\prime}(\phi)}{3 H(1+Q)}, \\
& \rho_{\mathrm{rad}}=C_{\gamma} T^{4} \approx \frac{\Gamma}{4 H} \dot{\phi}^{2},
\end{aligned}
$$

where $C_{\gamma}=\pi^{2} g_{\star} / 30$ is the Stefan-Boltzmann constant, while $g_{\star}$ denotes the number of degrees of freedom of the photon field. To obtain Eq. (51) we also used the approximations $\dot{\rho}_{\text {rad }} \ll H \rho_{\text {rad }}$, and $\dot{\rho}_{\text {rad }} \ll \Gamma \dot{\phi}^{2}$, respectively.

As an indicator of the accelerating, inflationary type behavior, we introduce the deceleration parameter $q$, defined as

$q=\frac{\mathrm{d}}{\mathrm{d} t} \frac{1}{H}-1$.

Negative values of $q$ indicate accelerating expansion, while positive values of the deceleration parameter correspond to decelerating cosmological dynamics. With the use of 
Eqs. (45) and (46) we immediately obtain for the deceleration parameter of the standard warm inflation theory the expression

$q=\frac{1}{2}\left[1+\frac{3\left(p_{\phi}+p_{\mathrm{rad}}\right)}{\rho_{\phi}+\rho_{\mathrm{rad}}}\right]$.

Comparable information as the one contained in the deceleration parameter $q$ can be obtained from the quantity $\epsilon_{H} \equiv$ $d \ln H / \mathrm{d} \mathcal{N}$, where $\mathcal{N}$ is the number of e-folds. Note that $\epsilon_{H}$ is related to the parameter $\epsilon$, which provides a useful description of the validity and properties of the slow-roll approximation in inflationary scenarios.

3.2 Gravitational field equations of the two non-comoving fluid cosmological model

For the scalar field-radiation two-fluid energy-momentum tensor, with components given by Eq. (24), the Einstein gravitational field equations can be written as [157]

$$
\begin{aligned}
& R_{\mu \nu} V^{\mu} V^{\nu}=\frac{1}{2}(\varepsilon+2 \Pi+\Psi), \\
& R_{\mu \nu} V^{\mu} h_{\sigma}^{\nu}=0, \\
& R_{\mu \nu} h_{\sigma}^{\mu} h_{\lambda}^{\nu}=\frac{1}{2}\left[\varepsilon-\frac{1}{3}(2 \Pi+\Psi)\right] h_{\sigma \lambda} \\
& \quad+(\Psi-\Pi)\left(\chi_{\sigma} \chi_{\lambda}-\frac{1}{3} h_{\sigma \lambda}\right),
\end{aligned}
$$

where $h^{\mu \nu}=g^{\mu \nu}-V^{\mu} V^{\nu}$. The conservation of the energymomentum tensor $\nabla_{\mu} T_{\nu}^{\mu}=0$ yields the equation

$$
\begin{aligned}
& \dot{\varepsilon}+(\varepsilon+\Pi) \nabla_{\mu} V^{\mu}-(\Psi-\Pi) \chi^{\prime \mu} V_{\mu}=0, \\
& (\varepsilon+\Pi) \dot{V}^{\mu}+h^{\mu \nu} \nabla_{\nu} \Pi+(\Psi-\Pi)^{\prime} \chi^{\mu} \\
& \quad+(\Psi-\Pi)\left(\nabla_{\nu} \chi^{\nu} \chi^{\mu}+\chi_{\nu}^{\prime} h^{\nu \mu}\right)=0,
\end{aligned}
$$

where the overdot and the prime are defined according to ( ) $=V^{\mu} \nabla_{\mu}(\text { ), and ( ) })^{\prime}=\chi^{\mu} \nabla_{\mu}($ ), respectively.

\subsubsection{Field equations for a Bianchi Type I geometry}

In the following, we make the simplifying assumption that on a large scale the very early Universe was homogeneous, and therefore all the cosmological quantities (four-velocities, energy densities, pressures, etc.) were functions of the cosmological time $t$ only. The assumption of homogeneity of the early Universe permits the introduction of a frame that is "comoving" with the auxiliary quantities $V$ and $\chi$. In the Cartesian coordinate system with $x^{0}=t, x^{1}=x$, $x^{2}=y$, and $x^{3}=z$, we may rescale the components of the four-velocities $V^{\mu}$ and $\chi^{\mu}$ as $V^{1}=V^{2}=V^{3}=0$, $V^{0} V_{0}=1$, and $\chi^{0}=\chi^{1}=\chi^{2}=0, \chi^{3} \chi_{3}=-1$, respectively $[155,157,158]$. Therefore, in the frame comoving with $V^{\mu}$ and $\chi^{\mu}$, the components of the energy-momentum tensor of the non-comoving scalar field and radiation fluids take the form

$T_{0}^{0}=\varepsilon, \quad T_{1}^{1}=T_{2}^{2}=-\Pi, \quad T_{3}^{3}=-\Psi$,

corresponding to an anisotropic fluid, where $\varepsilon$ is the total energy density of the mixture of fluids, $\Pi=P_{x}=P_{y}$ is the pressure along the $x$ and $y$ directions, while $\Psi=P_{z}$ is the total cosmological pressure along the $z$ direction. Since the non-comoving two-fluid scalar field-photon gas cosmological mixture represents an anisotropic fluid, on a cosmological scale the spacetime geometry must also be anisotropic. The components of the energy-momentum tensor, given by Eqs. (59), show that along the z-direction the total pressure $\Psi$ is generally different as compared with the pressure $\Pi$ exerted on the $x-y$ plane.

In a homogeneous Universe containing a scalar fieldradiation fluid mixture, the simplest geometry displaying this symmetry of the energy-momentum tensor is the spatially flat Bianchi Type I geometry, with the line element written as

$\mathrm{d} s^{2}=\mathrm{d} t^{2}-a_{1}^{2}(t) \mathrm{d} x^{2}-a_{2}^{2}(t) \mathrm{d} y^{2}-a_{3}^{2}(t) \mathrm{d} z^{2}$,

where $a_{i}(t)(i=1,2,3)$ are the directional scale factors. In the following we introduce the notations

$V=a_{1} a_{2} a_{3}, \quad H_{i}=\frac{\dot{a}_{i}}{a_{i}}, \quad i=1,2,3$,

and

$H=\frac{1}{3}\left(\sum_{i=1}^{3} H_{i}\right)=\frac{\dot{V}}{3 V}$

An important observational parameter, the expansion parameter $\theta$, is given by $\theta=3 H$.

In the Bianchi Type I geometry, with line element given by Eq. (60), the gravitational field equations for the noncomoving scalar field-photon gas mixture take the form

$$
\begin{aligned}
& 3 \dot{H}+H_{1}^{2}+H_{2}^{2}+H_{3}^{2}=-\frac{1}{2}(\varepsilon+\Psi+2 \Pi), \\
& \frac{1}{V} \frac{\mathrm{d}}{\mathrm{d} t}\left(V H_{i}\right)=\frac{1}{2}(\varepsilon-\Pi), \quad i=1,2,
\end{aligned}
$$

and

$\frac{1}{V} \frac{\mathrm{d}}{\mathrm{d} t}\left(V H_{3}\right)=\frac{1}{2}(\varepsilon-\Psi)$.

From the conservation of the total energy-momentum tensor of the scalar field-radiation fluid system, we obtain the evolution equation of the energy density of the warm inflationary model, given by

$\dot{\varepsilon}+3(\varepsilon+\Pi) H-(\Psi-\Pi) H_{3}=0$. 
By adding Eqs. (64) and (65) we immediately obtain

$\dot{H}+3 H^{2}=\frac{1}{2} \varepsilon-\frac{1}{3}\left(\frac{\Psi}{2}+\Pi\right)$,

or, equivalently,

$\ddot{V}+\left(\Pi+\frac{1}{2} \Psi-\frac{3}{2} \varepsilon\right) V=0$

3.3 Warm inflation with non-comoving cosmological fluids

By using the approximate expression (38) for the total energy density of the non-comoving scalar field and radiation, we obtain the conservation equation in the cosmologically expanding anisotropic scalar field-radiation mixture:

$$
\begin{aligned}
& \dot{\rho}_{\phi}+\dot{\rho}_{\mathrm{rad}}+3 H\left(\rho_{\phi}+p_{\phi}+\rho_{\mathrm{rad}}+p_{\mathrm{rad}}+2 F\right) \\
& \quad+\dot{F}-F H_{3}=0 .
\end{aligned}
$$

We assume now, similarly to the standard warm inflationary scenario, that the scalar field decays into radiation. Accordingly, we can reformulate Eq. (69) so that it describes the energy transfer from scalar field to radiation as two separate balance equations,

$\dot{\rho}_{\phi}+3 H\left(\rho_{\phi}+p_{\phi}\right)=-2 \dot{F}-12 F H=-\Gamma_{\phi}$,

$\dot{\rho}_{\text {rad }}+3 H\left(\rho_{\text {rad }}+p_{\text {rad }}\right)=\dot{F}+6 F H+F H_{3}=\Gamma_{\text {rad }}$,

where $\Gamma_{\phi}$ and $\Gamma_{\text {rad }}$ are the scalar field and radiation decay and creation rates, respectively. In order to build specific cosmological models we need to estimate the expression of $F\left(\rho_{\phi}, \rho_{\text {rad }}\right)$. In the following we will assume that the parameter $b$, described according to Eq. (29), the difference in the four-velocities of the radiation fluid and the scalar field is large, that is, $b \gg 1$. Consequently, at least at the initial stages of the cosmological expansion, the differences in the four-velocities of the two components of the fluid mixture were also large.

By assuming that $\rho_{\phi}+p_{\phi} \gg \rho_{\mathrm{rad}}+p_{\mathrm{rad}}$, from Eq. (31) we obtain for $b$ the expression

$b+2 \approx b \approx \sqrt{\frac{3}{4}} \frac{\dot{\phi}}{\sqrt{\rho_{\mathrm{rad}}}} \tan 2 \alpha$.

We would like to point out that the magnitude of $b$ is determined by the ratio $\dot{\phi} / \sqrt{\rho_{\text {rad }}}=\sqrt{2\left[\rho_{\phi}-U(\phi)\right] / \rho_{\text {rad }}}$, and the condition can also be satisfied for small values of $\alpha$, especially by taking into account the fact that at the beginning of the warm inflationary era the energy density of the radiation is small. Thus within the framework of these approximations we obtain first for $F$ the expression

$F \approx \frac{2}{3} b^{2} \rho_{\text {rad }}=\frac{1}{2} \dot{\phi}^{2} \tan ^{2} 2 \alpha$.
Now, by assuming that the angle $\alpha$ is small, we will approximate $\tan 2 \alpha \approx 2 \alpha$, thus obtaining

$F \approx 2 \alpha^{2} \dot{\phi}^{2}$,

where generally $\alpha$ is a function of the thermodynamic parameters of the scalar field and of the radiation fluid, $\alpha=$ $\alpha\left(\rho_{\phi}, p_{\phi}, \rho_{\mathrm{rad}}, p_{\mathrm{rad}}\right)$. Then, under these assumptions and simplifications the energy balance equations (70) and (71) take the form

$$
\begin{aligned}
& \ddot{\phi}+3 H\left[1+\frac{8 \alpha \dot{\alpha}}{3 H\left(1+8 \alpha^{2}\right)}\right] \dot{\phi}+\frac{U^{\prime}(\phi)}{1+8 \alpha^{2}}=0, \\
& \dot{\rho}_{\mathrm{rad}}+4 H \rho_{\mathrm{rad}}=4 \alpha^{2} \dot{\phi}^{2}\left(\frac{\dot{\alpha}}{\alpha}+\frac{\ddot{\phi}}{\dot{\phi}}\right) \\
& \quad+12 \alpha^{2} \dot{\phi}^{2} H+2 \alpha^{2} \dot{\phi}^{2} H_{3} .
\end{aligned}
$$

In order to obtain the full picture of the cosmological evolution we need to also solve the gravitational Einstein equations. For the considered form of the anisotropic energymomentum tensor from Eq. (64) it follows that we can assume $a_{1}(t)=a_{2}(t)$ without any loss of generality. The gravitational field equations (63)-(67) describing the non-comoving evolution of the scalar field-radiation fluid mixture take the form

$$
\begin{aligned}
3 \dot{H}+\sum_{k=1}^{3} H_{k}^{2} & =-\left[\left(1+2 \alpha^{2}\right) \dot{\phi}^{2}+\rho_{\mathrm{rad}}-U(\phi)\right], \\
\frac{1}{V} \frac{\mathrm{d}}{\mathrm{d} t}\left(V H_{i}\right) & =\alpha^{2} \dot{\phi}^{2}+U(\phi)+\frac{1}{3} \rho_{\mathrm{rad}}, i=1,2, \\
\frac{1}{V} \frac{\mathrm{d}}{\mathrm{d} t}\left(V H_{3}\right) & =U(\phi)+\frac{1}{3} \rho_{\mathrm{rad}} .
\end{aligned}
$$

The expansion parameter $\theta=3 H$ of the scalar fieldradiation fluid filled Universe is obtained:

$\theta=2 \frac{\dot{a}_{1}}{a_{1}}+\frac{\dot{a}_{3}}{a_{3}}$.

The shear scalar $\sigma$ of the anisotropic Universe is given by

$\sigma=\frac{1}{\sqrt{3}}\left(\frac{\dot{a}_{3}}{a_{3}}-\frac{\dot{a}_{1}}{a_{1}}\right)$.

With the use of Eqs. (80) and (81) it is possible to determine the directional Hubble parameters $H_{1}$ and $H_{3}$ in terms of observable cosmological parameters as

$H_{1}=\frac{\theta}{3}-\frac{1}{\sqrt{3}} \sigma, \quad H_{3}=\frac{\theta}{3}+\frac{2}{\sqrt{3}} \sigma$,

respectively. In terms of the observable quantities, the anisotropic cosmological pressure differences can be obtained:

$\Psi-\Pi=\sqrt{3} \sigma+\theta \sigma$. 
The volume evolution equation (68) can be written as

$\ddot{V}-3\left(U(\phi)+\rho_{\text {rad }}+\frac{2}{3} \alpha^{2} \dot{\phi}^{2}\right) V=0$.

\section{The slow-roll approximation}

In the present section we are going to investigate the warm inflationary scenario introduced in the previous section by assuming both the quasi-stability and the slow-roll conditions. Besides these two well-known assumptions, it is usual to study the warm inflationary models in two important limits, namely the weak and the strong dissipative regimes, respectively. Here first we obtain the general forms of the relevant equations describing the warm inflationary regime. Then we will discuss in detail the asymptotic regimes of the inflationary behavior in the presence of non-comoving motions of radiation and scalar field.

\subsection{Evolution equations for non-comoving warm inflation}

We begin our investigation with the wave equation, i.e. Eq. (75), in which, by assuming the condition $\ddot{\phi} / \dot{\phi} \ll 3 H$, the time variation $\dot{\phi}$ of the inflaton field can be expressed as

$\dot{\phi}=-\frac{U^{\prime}(\phi)}{3 H(1+Q)\left(1+8 \alpha^{2}\right)}$,

where

$Q=\frac{\Gamma}{3 H}=\frac{8 \alpha \dot{\alpha}}{3 H\left(1+8 \alpha^{2}\right)}$

gives the dissipation function, while, as already indicated in Eq. (49), $\Gamma$ is the dissipation coefficient. Now whereas we are working in a quasi-stable environment, with $\dot{\rho}_{\text {rad }} \ll H \rho_{\text {rad }}$, Eq. (76) can be expressed as follows:

$4 H \rho_{\mathrm{rad}}=4 \alpha^{2} \dot{\phi}^{2}\left(\frac{\dot{\alpha}}{\alpha}+3 H+\frac{H_{3}}{2}\right)$.

From Eqs. (78) and (79) one obtains immediately for the term $\alpha^{2} \dot{\phi}^{2}$ the expression

$\alpha^{2} \dot{\phi}^{2}=\frac{1}{V} \frac{\mathrm{d}}{\mathrm{d} t}\left(V H_{1}\right)-\frac{1}{V} \frac{\mathrm{d}}{\mathrm{d} t}\left(V H_{3}\right)$.

To find an explicit expression of the above equations, and without losing any generality, one can assume that $a_{3}(t)=$ $a_{i}^{\lambda}(t), i=1,2$, in which $\lambda$ is a constant whose value should be obtained from the comparison with the observational data [170-172]. Since the scale factor for the $x$ direction is the same as for the $y$ one, so that $a_{1}(t)=a_{2}(t)$, consequently, from Eqs. (61) and (62) we obtain at once

$V=a_{i}^{2+\lambda}, \quad H_{3}=\lambda \frac{\dot{a}_{i}}{a_{i}}, \quad i=1,2$, and

$H=\frac{1}{3}(2+\lambda) H_{i}=\frac{\dot{V}}{3 V}, \quad i=1,2$,

respectively. By combining Eqs. (88)-(90), it follows that the term $\alpha^{2} \dot{\phi}^{2}$ can be expressed as follows:

$\alpha^{2} \dot{\phi}^{2}=(1-\lambda)\left[(2+\lambda) H_{i}^{2}+\dot{H}_{i}\right], \quad i=1,2$.

Next in Eq. (87) we suppose that

$\frac{\dot{\alpha}}{\alpha}=-h_{0} H_{i}, \quad i=1,2$,

where $h_{0}$ is a constant that can be constrained by the data.

To determine $\alpha$ we can use the generalized de Sitter scale factor, the so-called intermediate scale factor [168-172], and a power-law expression of the scale factor. As the first example we can consider $a_{i}(t)=a_{0 i} e^{\gamma t^{n}}$, while for the power-law case we will introduce the scale factor as $\bar{a}_{i}(t)=\bar{a}_{0 i} t^{m}$ (see [173] and the references therein). Here $a_{0 i}, \bar{a}_{0 i}, n$ and $m$ are some constants that will be fixed by means of observations. From Eq. (92), and for the generalized de Sitter and power-law cases, one finds

$\alpha=\alpha_{0} e^{-h_{0} \gamma t^{n}}=\alpha_{0}\left(\frac{a_{i}(t)}{a_{0 i}}\right)^{-h_{0}}, \quad i=1,2$,

and

$\alpha=\bar{\alpha}_{0} t^{-h_{0} m}=\bar{\alpha}_{0}\left(\frac{\bar{a}_{i}(t)}{\bar{a}_{0 i}}\right)^{-h_{0}}, \quad i=1,2$,

where $\alpha_{0}$ and $\bar{\alpha}_{0}$ are some arbitrary integration constants. From the above equations and from Eq. (91) the expression of the inflaton field as a function of the cosmic time can be straightforwardly obtained. Thus, for the generalized de Sitter and power-law scale factors, respectively, it follows that

$\dot{\phi}^{2}=\frac{n \gamma(1-\lambda)}{\alpha_{0}^{2} e^{-2 h_{0} \gamma t^{n}}}\left[(2+\lambda) t^{n}-n \gamma(1-n)\right] t^{n-2}$,

and

$\dot{\phi}^{2}=\frac{m(1-\lambda)}{\bar{\alpha}_{0}^{2}}[(2+\lambda) m-1] t^{-2\left(1-h_{0} m\right)}$.

In the warm inflationary scenarios, the slow-roll approximations are still valid, that is, the rate of the Hubble parameter during a Hubble time is assumed to be smaller than unity. This condition is imposed via the first slow-roll parameter

$\epsilon_{1}=-\frac{\dot{H}}{H^{2}}$

which satisfies the constraint $\epsilon_{1} \ll 1$. Besides the quasi-de Sitter assumption leading to Eq. (97), the energy density of the inflaton field dominates over the radiation energy density. 
Moreover, the kinetic term of the scalar field is negligible as compared to its potential, i.e. $\rho_{\phi} \gg \rho_{\text {rad }}$ and $\rho_{\phi} \simeq U(\phi)$.

Then, from Eqs. (51), (67), (87), (90), and (92), it follows that

$$
\begin{aligned}
& 3 H^{2}=\frac{(2+\lambda)^{2}}{3} H_{i}^{2} \simeq U(\phi), \quad i=1,2, \\
& \rho_{\mathrm{rad}}:=C_{\gamma} T^{4}=\left(\frac{3}{2+\lambda}\right)\left(\frac{2 h_{0}+3 \lambda+4}{2}\right) \alpha^{2} \dot{\phi}^{2},
\end{aligned}
$$

where $T$ is the temperature of the photon gas. By substituting Eq. (97) into (98), and with the use of Eq. (85), it follows that in the warm inflationary scenario with non-comoving scalar field and radiation the slow-roll parameters can be expressed as

$$
\epsilon_{1}=\frac{1}{2(1+Q)} \frac{U^{\prime 2}(\phi)}{\left(1+8 \alpha^{2}\right) U^{2}(\phi)}, \quad \epsilon_{2}=\frac{\dot{\epsilon}_{1}}{H \epsilon_{1}} .
$$

In an isotropic background, i.e. $\lambda=1$, and when $\alpha$ tends to zero, the first slow-roll parameter goes back to the usual warm inflation relation $[138,174,175]$. To examine the validity of a theoretical model, one basic approach is to compare its predictions with the observations. In doing so for the non-comoving warm inflationary model, at first we will obtain some important perturbations parameters, including the amplitude of the scalar perturbations, $\mathcal{P}_{S}$, the amplitude of the tensor perturbations $\mathcal{P}_{t}$, the scalar and tensor spectral indices $n_{s}, n_{t}$, and the tensor-to-scalar ratio $r$, respectively. Then we will compare the predictions of the theoretical model with the Planck 2018 data. Following $[85,87,103,119]$, the amplitude of the scalar perturbations is calculated to be

$\mathcal{P}_{s}=\left(\frac{H^{2}}{2 \pi \dot{\phi}}\right)^{2}\left(1+2 n_{B E}+\frac{2 \sqrt{3} \pi Q}{\sqrt{3+4 \pi Q}} \frac{T}{H}\right) G(Q)$,

where $n_{B E}$ is the Bose-Einstein distribution, given by $n_{B E}=$ $\left[\exp \left(H / T_{\delta \phi}\right)-1\right]^{-1}$, and $T_{\delta \phi}$ is the temperature of the inflaton fluctuations [119]. Here $G(Q)$, which describes the growth of the fluctuations, is a function of $Q$, and its presence has arisen from the coupling of the scalar field and radiation [103,119].

The scalar spectral index and its running behavior, $\alpha_{s}$, are obtained from the amplitude of the scalar perturbations, and they are defined as

$n_{s}-1=\frac{\mathrm{d} \ln \left(\mathcal{P}_{s}\right)}{\mathrm{d} \ln (k)}, \quad \alpha_{s}=\frac{\mathrm{d} n_{s}}{\mathrm{~d} \ln (k)}$.

Tensor perturbations, representing the gravitational waves, are measured indirectly through the tensor-to-scalar ratio parameter $r=\mathcal{P}_{t} / \mathcal{P}_{s}$. The amplitude of the tensor perturbations is given by [103]

$\mathcal{P}_{t}=\frac{2 H^{2}}{\pi^{2}}$

The tensor spectral index is defined as

$n_{t}=\frac{\mathrm{d} \ln \left(\mathcal{P}_{t}\right)}{\mathrm{d} \ln (k)}$

To measure the amount of cosmic expansion during inflation it is customary to use the number of e-folds $\mathcal{N}$, defined as

$\mathcal{N}=\int_{t_{\star}}^{t_{e}} H \mathrm{~d} t=\int_{\phi_{\star}}^{\phi_{e}} \frac{H}{\dot{\phi}} \mathrm{d} \phi$

where the subscript $\star$ denotes the horizon exit values.

In the warm inflationary approach, it is a common practice to investigate the model in two different and important thermal regimes, known as the strong and the weak dissipative regimes,respectively, where the dissipative ratio satisfies the conditions $Q \gg 1$, and $Q \ll 1$, respectively. From Eq. (100) it should be noted that inflation ends when

$\epsilon_{1}=1+Q$

where for the weak and the strong regimes $\epsilon_{1}$ behaves as $\epsilon_{1} \simeq 1$, and $\epsilon_{1} \simeq Q$, respectively [136]. In the subsequent subsections, we are going to investigate in detail the noncomoving warm inflationary model in the presence of scalar field and radiation in these two limiting regimes.

\subsection{Weak dissipative regime}

In the weak dissipative regime, the dissipative ratio is much smaller than unity, i.e., $Q \ll 1(\Gamma \ll 3 H)$. Consequently, we also have $(1+Q) \simeq 1$. Moreover, in this regime, the parameter $G(Q) \simeq 1$ at the time of horizon crossing. Then, from Eq. (101), the amplitude of the scalar perturbations in the weak regime reads

$\mathcal{P}_{s}=2 \mathcal{P}_{s 0}\left(\frac{H^{2}}{2 \pi \dot{\phi}}\right)^{2} \frac{T}{H}$,

where $\mathcal{P}_{s 0}$ is a constant should be determined comparing to data. To investigate the consistency of the warm inflationary models one has to test the condition $T / H>1$, besides other observational constraints. Thus, for the case of the noncomoving warm inflation from Eqs. (85), (98) and (99) we obtain

$$
\frac{T}{H}=\left(\frac{\sqrt{T_{0}} \alpha}{1+8 \alpha^{2}}\right)^{\frac{1}{2}} \frac{U^{\prime \frac{1}{2}}}{U^{\frac{3}{4}}},
$$


Table 1 Estimation of the free parameters of the non-comoving warm inflationary model in the weak dissipation regime with power-law scale factors by using the Planck 2013, 2015 and 2018 data sets, and for
$C_{\gamma}=50$. To obtain the presented results we have fixed the power spectrum to its horizon exit value, i.e., $\mathcal{P}_{s}=2.17 \times 10^{-9}$. For this case we restrict our analysis to real values of the scalar field at the horizon exit

\begin{tabular}{lllllllllll}
\hline$m$ & $\lambda$ & $\phi_{\star}$ & $\phi_{\mathrm{e}}$ & $h_{0}$ & $\bar{\alpha}_{0}$ & $n_{\mathrm{s}}$ & $r_{\star}$ & $\mathcal{P}_{s 0}$ & $T\left(\phi_{\star}\right) / H\left(\phi_{\star}\right)$ & $\mathcal{N}$ \\
\hline 29.950 & 11.810 & 1809.87 & 3169.22 & 0.0096592 & 26.1 & 0.944287 & 0.0000111051 & 2.96471 & 1.05881 & 58 \\
29.952 & 11.812 & 1792.78 & 3169.75 & 0.0096591 & 26.02 & 0.938879 & 0.0000111068 & 2.52561 & 1.01903 \\
29.949 & 11.814 & 1793.13 & 3170.36 & 0.00965899 & 26.04 & 0.964083 & 0.0000110715 & 2.56107 & 1.02204 \\
29.948 & 11.816 & 1810.85 & 3170.9 & 0.00965898 & 26.06 & 0.968554 & 0.0000111356 & 2.92386 & 1.05495 \\
\hline
\end{tabular}

where

$T_{0}=\frac{3^{2}}{(2+\lambda) C_{\gamma}}\left(\frac{2 h_{0}+3 \lambda+4}{2}\right)$.

Hence the ratio of the photon temperature and of the Hubble function depends on the function $\alpha$, describing the differences in the four-velocities of the radiation and scalar field.

From Eq. (102), and from the combination of Eqs. (107) and (108), the scalar spectral index is obtained:

$n_{s}-1=-\frac{9}{2} \epsilon_{1}+3 \eta-\beta$

where

$\epsilon_{1}=\frac{1}{2\left(1+8 \alpha^{2}\right)} \frac{U^{\prime 2}(\phi)}{U^{2}(\phi)}$

For the potential slow-roll parameter $\eta$ we find

$\eta=\frac{1}{2\left(1+8 \alpha^{2}\right)} \frac{U^{\prime \prime}(\phi)}{U(\phi)}$,

while the slow-roll parameter $\beta$ is given as

$\beta=\frac{1}{2\left(1+8 \alpha^{2}\right)} \frac{U^{\prime}(\phi) \Gamma_{\mathrm{eff}}^{\prime}}{U(\phi) \Gamma_{\mathrm{eff}}}$,

where $\Gamma_{\text {eff }}=\alpha\left(1+8 \alpha^{2}\right)^{3}$.

For the tensor-to-scalar ratio, from Eqs. (85), (103) and (107), in the weak dissipative regime, we also obtain

$r=\frac{8 \epsilon_{1}}{1+8 \alpha^{2}} \frac{H}{T}$

As one can see, all these important observational parameters are functions of $\alpha$, describing the deviations from the comoving motion of the fluid components of the early Universe.

\subsubsection{Weak dissipative regime with power-law scale factor}

In this subsection we are going to investigate the compatibility of the theoretical results of the non-comoving warm inflationary model obtained within the weak dissipative regime with power-law scale factor to the recent observational data. In doing so we will consider the Planck 2013, 2015, and 2018 data sets as our criteria.

From Eq. (96) a relation for the cosmic time as a function of the scalar field can be obtained:

$t(\phi)=\left[\frac{h_{0} \bar{\alpha}_{0} m}{\sqrt{m(1-\lambda)[(\lambda+2) m-1]}} \phi\right]^{\frac{1}{h_{0} m}}$.

By substituting Eq. (114) into Eqs. (85) and (96), for the weak regime with power-law scale factor, the scalar field potential can be obtained as a function of the scalar field as

$$
U(\phi)=\left[3 h_{0}^{2} m\left(1-m h_{0}\right) \phi^{2}+24(\lambda-1)\left(h_{0} m-1\right)\right.
$$

$$
\times(m \lambda+2 m-1)] \times \frac{h_{0} m^{3}\left[\frac{h_{0} m \phi}{\sqrt{-\frac{(\lambda-1) m((\lambda+2) m-1)}{\bar{\alpha}_{0}^{2}}}}\right]^{-\frac{3}{h_{0} m}} \phi}{\left(h_{0} m-3\right)\left(h_{0} m-1\right)} .
$$

In addition, by taking Eq. (110) equal to unity, and after some simple mathematical manipulations, we obtain the magnitude of the scalar field at the end of inflation as presented in Table 1.

Then, by using Eqs. (96) and (105), the scalar field at the horizon exit can be obtained in the non-comoving warm inflation model as

$\phi_{\star}=\phi_{e} \times \exp \left[-h_{0} \mathcal{N}\right]$

Now, by using Eq. (116), and the results of the Sect. 4.2, especially Eqs. (109) and (113), we can examine the behavior of the scalar spectral index versus the tensor-to-scalar ratio, and of the temperate-Hubble parameter ratio against the number of e-folds at the horizon exit in the non-comoving warm inflationary model.

The results of the comparison between the theoretical results and the observations can be summarized in Table 1, and Figs. 1 and 2, respectively. 


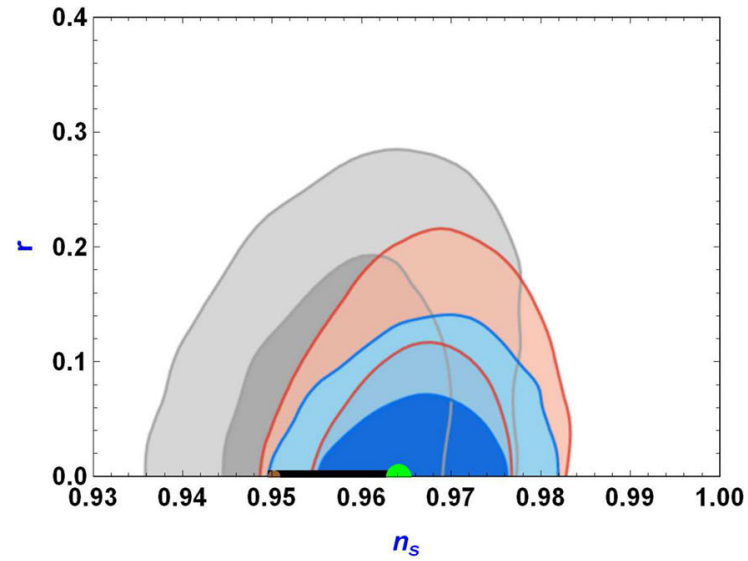

Fig. 1 The $r-n_{s}$ diagram comparing the predictions of the noncomoving warm inflation model in the weak regime with power-law scale factors, having the model parameters of Table 1, with the observational data of the Planck 2013, 2015 and 2018 data sets. In the left panel, the likelihood of Planck 2013 are indicated with gray contours, Planck TT+lowP with red contours, and Planck TT, TE, EE+lowP (2015) with

\subsubsection{Weak dissipative regime with generalized de Sitter scale factor}

Now we are going to investigate the compatibility of the theoretical predictions of the non-comoving warm inflationary model, obtained within the weak dissipative regime, with generalized de Sitter scale factor, to the recent observational data. In doing so we will consider the Planck 2013, 2015, and 2018 data sets as our criteria.

From Eq. (95), the relation of the cosmic time as a function of the scalar field can be obtained:

$t(\phi)=-\frac{1}{2 \gamma h_{0}} \ln \left[-\frac{\lambda^{2}+\lambda-2}{\gamma\left(\alpha_{0} h_{0} \phi\right)^{2}}\right]$.

By substituting Eq. (117) into Eqs. (85) and (95), for the weak regime with generalized de Sitter case, the scalar field potential as a function of the scalar field reads

$U(\phi)=\frac{3 \gamma\left[8\left(-\lambda^{2}-\lambda+2\right) \ln \left(-\frac{\lambda^{2}+\lambda-2}{\alpha_{0}^{2} \gamma h_{0}^{2} \phi^{2}}\right)+\gamma h_{0}^{2} \phi^{2}\right]}{2 h_{0}}$.

In addition, by taking Eq. (110) equal to unity, and after some simple mathematical manipulations, we obtain the magnitude of the scalar field at the end of inflation as presented in Table 2.

Then, by using Eqs. (95) and (105), the scalar field at the horizon exit can be obtained in the non-comoving warm

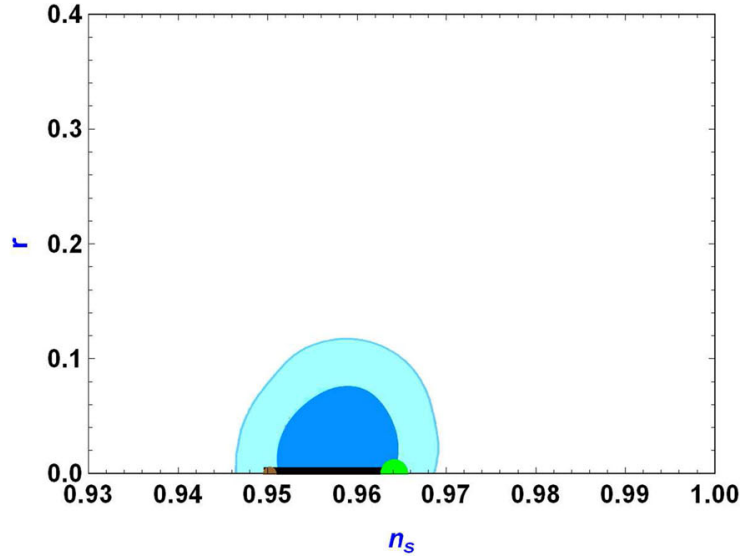

blue contours. In the right panel, the results of Planck 2018 are indicated by dark and light blue colors, referring to $68 \%$ and $95 \%$ confidence levels, respectively. In both figures the thick black lines give the predictions of the non-comoving warm inflation model, while the small, brown, and large, green, circles are the values of $n_{\mathrm{s}}$ at the number of e-folds $\mathcal{N}=58$ and $\mathcal{N}=62$, respectively

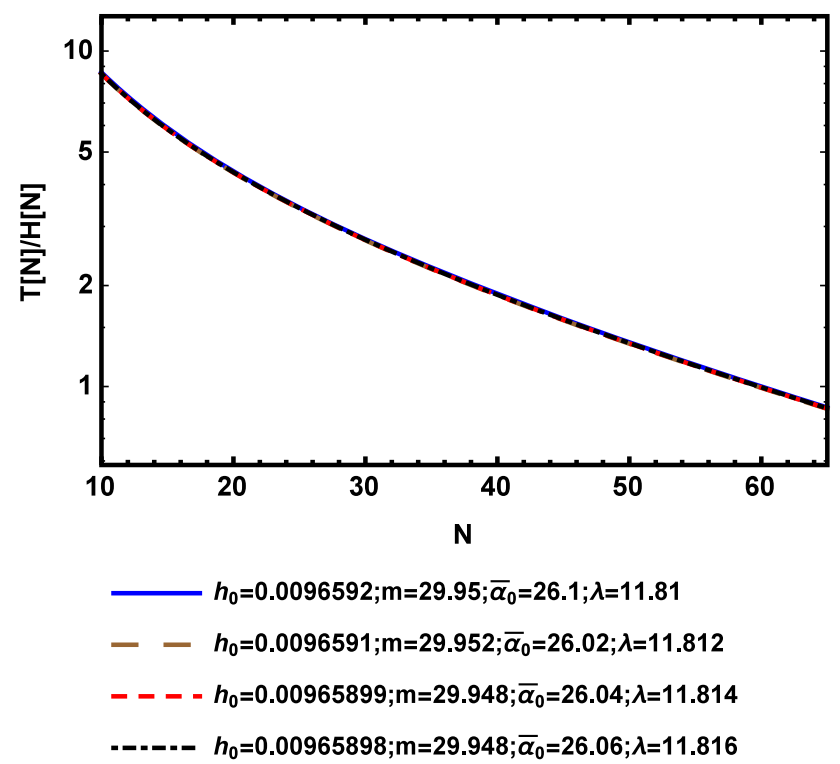

Fig. 2 The ratio of the temperature to the Hubble parameter during the inflationary evolution of the non-comoving warm inflationary model in the weak dissipation regime with power-law scale factors versus the number of e-folds, for different values of parameters of the model. During inflation the temperature is higher than the Hubble parameter, and their ratio decreases when approaching the end of inflation

inflation model as

$\phi_{\star}=\phi_{e} \times \exp \left[-h_{0} \mathcal{N}\right]$.

Now, by using Eq. (119), and by taking into account the results of Sect. 4.2, especially Eqs. (109) and (113), we can investigate the behavior of the scalar spectral index versus the tensor-to-scalar ratio, and of the temperate-Hubble parameter 
Table 2 Estimation of the free parameters of the non-comoving warm inflationary model in the weak dissipation regime with generalized de Sitter scale factors by using the Planck 2013, 2015 and 2018 data sets, and for $C_{\gamma}=55$. To obtain the presented values of the model param- eters we have fixed the power spectrum to its horizon exit value, i.e., $\mathcal{P}_{s}=2.17 \times 10^{-9}$. For this case we restrict our analysis to real values of the scalar field at the horizon exit

\begin{tabular}{lllllllllll}
\hline$\gamma$ & $\lambda$ & $\left|\phi_{\star}\right|$ & $\left|\phi_{\mathrm{e}}\right|$ & $h_{0}$ & $\alpha_{0}$ & $n_{\mathrm{s}}$ & $r_{\star} \times 10^{-7}$ & $\mathcal{P}_{s 0} \times 10^{-20}$ & $T\left(\phi_{\star}\right) / H\left(\phi_{\star}\right)$ & $\mathcal{N}$ \\
\hline 33 & 0.02 & 0.00369826 & 1.58425 & 0.101 & 60 & 0.959775 & 4.96773 & 1.26815 & 3.3729 \\
34 & 0.03 & 0.00351334 & 1.5981 & 0.102 & 61 & 0.958454 & 4.76646 & 1.19188 & 3.36302 \\
35 & 0.04 & 0.00334193 & 1.61412 & 0.103 & 62 & 0.956301 & 4.73632 & 1.13156 & 3.35017 \\
36 & 0.05 & 0.00317758 & 1.62965 & 0.104 & 63 & 0.954101 & 4.69175 & 1.07226 & 3.33757 \\
\hline
\end{tabular}

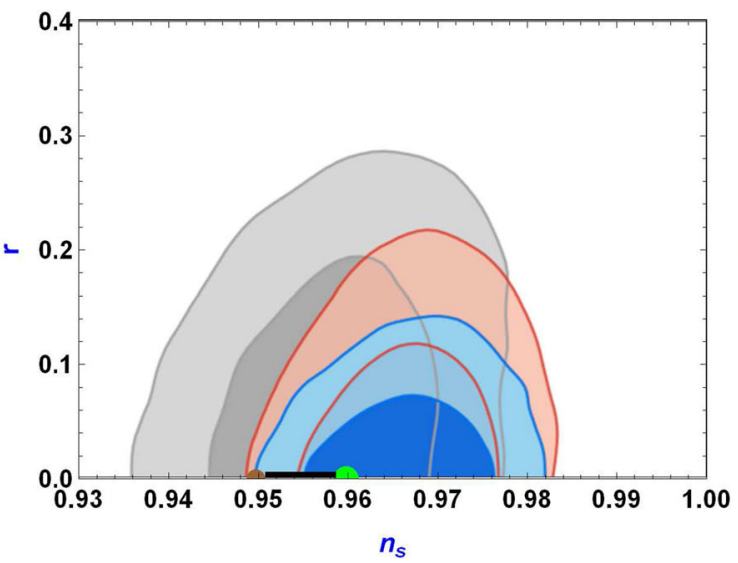

Fig. 3 The $r-n_{s}$ diagram comparing the predictions of the noncomoving warm inflation model in the weak regime with generalized de Sitter scale factors, having the model parameters of Table 1, with the observational data of the Planck 2013, 2015 and 2018 data sets. In the left panel, the likelihood of Planck 2013 are indicated with gray contours, Planck TT+lowP with red contours, and Planck TT,TE,EE+lowP

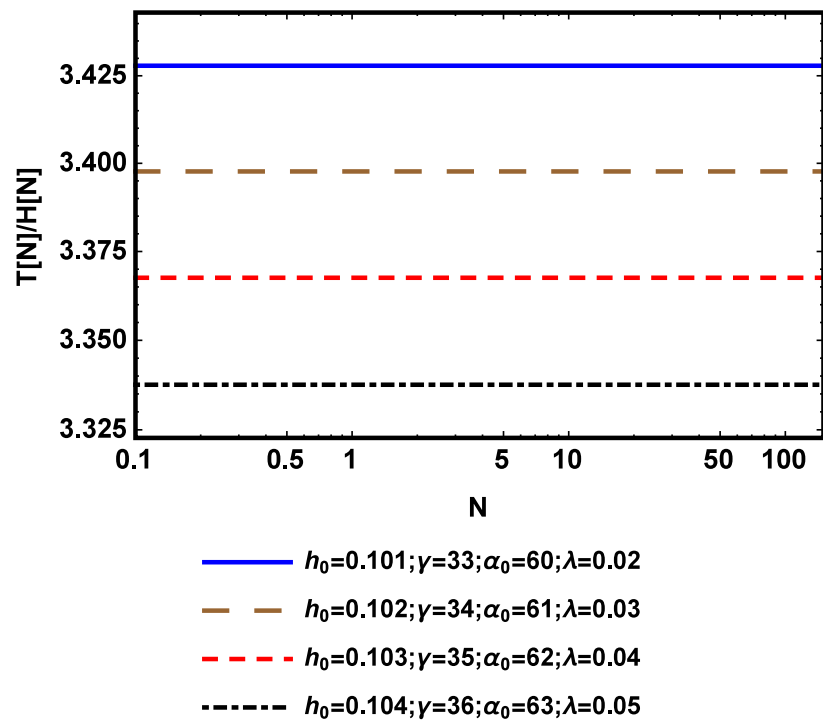

Fig. 4 The ratio of the temperature to the Hubble parameter during the inflationary evolution of the non-comoving model in the weak regime with generalized de Sitter scale factors versus the number of e-folds, for different values of the parameters of the model

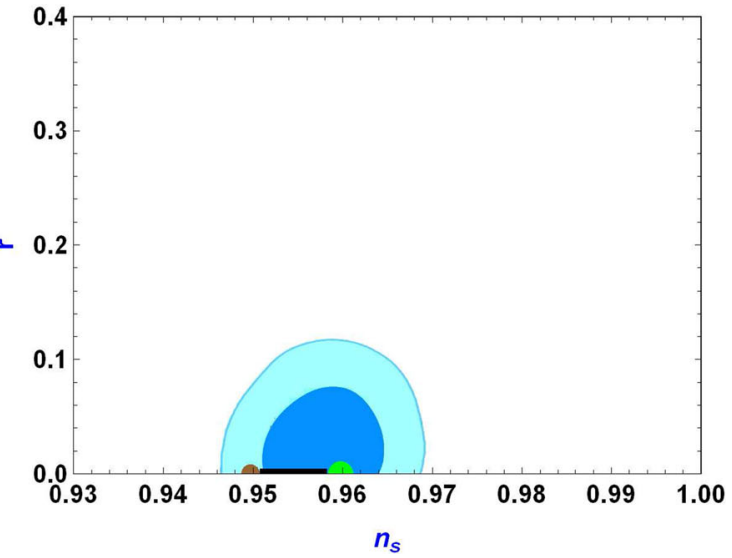

(2015) with blue contours. In the right panel, the results of Planck 2018 are indicated by dark and light blue colors, referring to $68 \%$ and $95 \%$ confidence levels, respectively. In both figures the thick black lines give the predictions of the non-comoving warm inflation model, while the small, brown, and large, green, circles are the values of $n_{\mathrm{s}}$ at the number of e-folds $\mathcal{N}=58$ and $\mathcal{N}=62$, respectively

ratio against the number of e-folds at the horizon exit for the non-comoving warm inflationary mode with generalized de Sitter scale factor.

The results of the comparison between the theoretical results and the observations have been summarized in Table 2 and Figs. 3 and 4.

\subsection{Strong dissipative regime}

In the strong dissipative regime, Eq. (101) takes the form

$\mathcal{P}_{s}=\left(\frac{H^{2}}{2 \pi \dot{\phi}}\right)^{2} \sqrt{3 \pi Q} \frac{T}{H} \times G(Q)$.

In the following we will consider models in which the dissipation coefficient is introduced phenomenologically via an ansatz of the form $\Gamma=\Gamma_{0} T^{\zeta} / \phi^{\zeta-1}$, where $\Gamma_{0}$ and $\zeta$ are constants $[103,119,137,175]$. Then the function $G(Q)$, depending on the different values of the parameter $\zeta$, can be 
approximated by [103,119]

$$
\begin{aligned}
\zeta & =1 \longrightarrow G(Q) \simeq 1+0.127 Q^{4.330}+4.981 Q^{1.946} \\
\zeta=3 \longrightarrow G(Q) & \simeq 1+0.0185 Q^{2.315}+0.335 Q^{1.364} \\
\zeta=-1 \longrightarrow G(Q) & \simeq \frac{1+0.4 Q^{0.77}}{\left(1+0.15 Q^{1.09}\right)^{2}} .
\end{aligned}
$$

Hence all the necessary parameters required to study the evolution of a warm inflationary evolution come from the above expressions of the decay rate $\Gamma$. However, there is no need to restrict our study to only these three parameters. But, without any loss of generality, and in order to check the compatibility of the non-comoving warm inflationary model with the observations, following [137], we only consider one of the $\zeta$ values per each case.

Therefore, in a more convenient way, and by taking into account that $Q \gg 1$, we can write down the function $G(Q)=$ $a_{\zeta} Q^{b_{\zeta}}$, where

$$
\begin{aligned}
\zeta=1 \longrightarrow a_{\zeta} & =0.127, \quad b_{\zeta}=4.330, \\
\zeta=3 \longrightarrow a_{\zeta} & =0.0185, \quad b_{\zeta}=2.315, \\
\zeta=-1 \longrightarrow a_{\zeta} & =17.78, \quad b_{\zeta}=-1.41 .
\end{aligned}
$$

Hence the amplitude of the scalar perturbations is obtained:

$\mathcal{P}_{s}=\mathcal{P}_{s 0}\left(\frac{H^{2}}{2 \pi \dot{\phi}}\right)^{2} \sqrt{3 \pi Q} \frac{T}{H} \times a_{\zeta} Q^{b_{\zeta}}$.

For the strong dissipation case, by taking into account Eqs. (85), (98), and (99), we obtain

$$
\frac{T}{H}=\left(\frac{3 \sqrt{T_{0}} \alpha}{1+8 \alpha^{2}}\right)^{\frac{1}{2}} \frac{U^{\prime \frac{1}{2}}}{\Gamma^{\frac{1}{2}} U^{\frac{1}{2}}}
$$

where $T_{0}$ is the same as in Eq. (108). By taking the time derivative of Eq. (121), and using the definitions of Eq. (102), leads to the scalar spectral index in the strong dissipative regime of the non-comoving warm inflation as given by

$n_{s}-1=\left(b_{\zeta}-\frac{5}{2}\right) \epsilon_{1}+3 \eta-4 \beta-2 b_{\zeta} \beta_{1}$,

where

$\epsilon_{1}=\frac{1}{2 Q\left(1+8 \alpha^{2}\right)} \frac{U^{\prime 2}(\phi)}{U^{2}(\phi)}$.

For the potential slow-roll parameter $\eta$ we find

$\eta=\frac{1}{2 Q\left(1+8 \alpha^{2}\right)} \frac{U^{\prime \prime}(\phi)}{U(\phi)}$.
The slow-roll parameters $\beta$ and $\beta_{1}$ are given by

$\beta=\frac{1}{2 Q\left(1+8 \alpha^{2}\right)} \frac{U^{\prime}(\phi) \Gamma^{\prime}}{U(\phi) \Gamma}$

and

$\beta_{1}=\frac{1}{2 Q\left(1+8 \alpha^{2}\right)} \frac{U^{\prime}(\phi) \Gamma_{\mathrm{eff}}^{\prime}}{U(\phi) \Gamma_{\mathrm{eff}}}$,

where $\Gamma_{\text {eff }}=\alpha \Gamma$. We notice that the function $G(Q)$ for $\zeta=1$ and $\zeta=3$ has an acceptable behavior for the value $\zeta=-1$, or even for $\zeta=1$.

The amplitude of the tensor perturbations in the strong dissipative regimes is given by $[103,119]$

$\mathcal{P}_{t}=\frac{2 H^{2}}{\pi^{2}}$

Then, from Eqs. (120), (124) and (128), the tensor-toscalar ratio reads

$r=\frac{16 \epsilon_{1} \sqrt{Q}}{\sqrt{3 \pi}\left(1+8 \alpha^{2}\right) G(Q)} \frac{H}{T}$.

\subsubsection{Strong dissipative regime with power-law scale factor}

Following the procedure of Sect. 4.2, we are going to investigate the accuracy of the non-comoving warm inflation model with power-law scale factor in the strong regime. First, by substituting Eq. (114) into Eqs. (85) and (96), for the strong regime with power-law scale factor the scalar field potential as a function of the scalar field is expressed as

$$
\begin{aligned}
U(\phi)= & 4 h_{0}(\lambda-1) m^{2}[(\lambda+2) m-1] \\
& \times\left\{\frac{h_{0} m \phi}{\sqrt{-\frac{(\lambda-1) m[(\lambda+2) m-1]}{\bar{\alpha}_{0}^{2}}}}\right\}^{-\frac{2}{h_{0} m}} .
\end{aligned}
$$

Also from Eqs. (130) and (86), for the dissipation ratio $Q$ one obtains

$Q(\phi)=-\frac{8\left(\frac{\bar{\alpha}_{0} h_{0} m \phi}{\sqrt{(\lambda-1)(-m)((\lambda+2) m-1)}}\right)^{\frac{1}{h_{0} m}}}{3 m \phi\left(8-\frac{\mathrm{h} 0^{2} m \phi^{2}}{(\lambda-1)((\lambda+2) m-1)}\right)}$.

Now we want to test the theoretical predictions of the noncomoving warm inflationary model in the strong dissipative regime by comparing the theoretical results to the observational data sets. In order to perform such a comparison, at first and by means of Eqs. (106) and (124) for the end of inflation values of the scalar field, we obtain the results expressed in Table 3. 
Table 3 The estimations of the free parameters of the non-comoving warm inflationary model in the strong dissipation regime with powerlaw scale factor by using the Planck 2013, 2015 and 2018 data sets, and for $C_{\gamma}=50$. We have restricted our analysis to real values of the scalar field at the horizon exit. To obtain the presented results we have fixed the power spectrum to its horizon exit value, i.e., $\mathcal{P}_{s}=2.17 \times 10^{-9}$, and we have used $a_{\zeta}=0.127$, and $b_{\zeta}=4.330$, respectively

\begin{tabular}{|c|c|c|c|c|c|c|c|c|c|c|}
\hline$m$ & $\lambda$ & $\phi_{\star}$ & $\phi_{\mathrm{e}}$ & $h_{0}$ & $\bar{\alpha}_{0}$ & $n_{\mathrm{s}}$ & $r_{\star}$ & $\mathcal{P}_{s 0} \times 10^{-16}$ & $T\left(\phi_{\star}\right) / H\left(\phi_{\star}\right)$ & $\mathcal{N}$ \\
\hline 7.79931 & 217 & 0.102944 & 13.8467 & 0.100033 & 188 & 0.954748 & 0.0892411 & 8.48726 & 1.00639 & 50 \\
\hline 7.79930 & 218 & 0.101816 & 13.6944 & 0.100032 & 189 & 0.954852 & 0.087325 & 8.05104 & 1.00316 & 50 \\
\hline 7.79929 & 216 & 0.106635 & 14.3446 & 0.100035 & 186 & 0.954644 & 0.0945849 & 9.73916 & 1.02192 & 50 \\
\hline 7.79928 & 215 & 0.107853 & 14.5091 & 0.100036 & 185 & 0.954538 & 0.0967116 & 10.02805 & 1.02537 & 50 \\
\hline
\end{tabular}

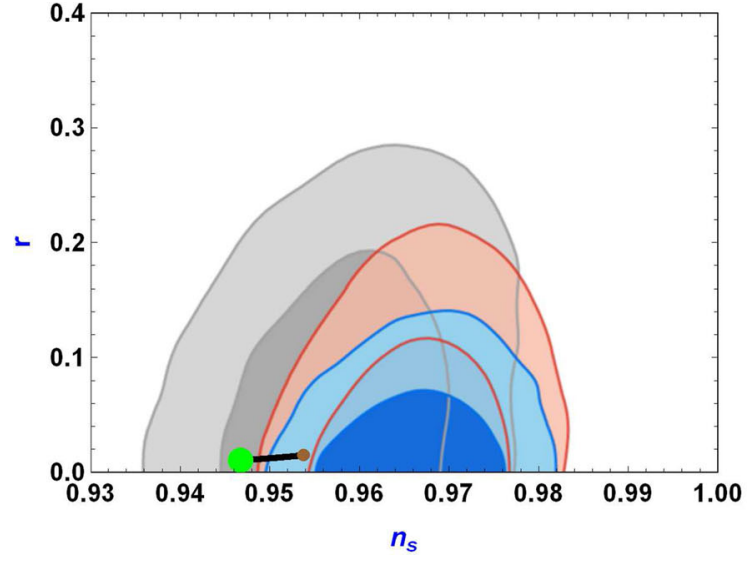

Fig. 5 The $r-n_{s}$ diagram comparing the predictions of the noncomoving warm inflation model in the strong dissipative regime with power-law scale factor and with the free parameters given in Table 3, with the Planck 2013, 2015 and 2018 data sets. In the left panel, the likelihood of Planck 2013 are indicated with gray contours, Planck TT+lowP with red contours, and Planck TT,TE,EE+lowP (2015) with

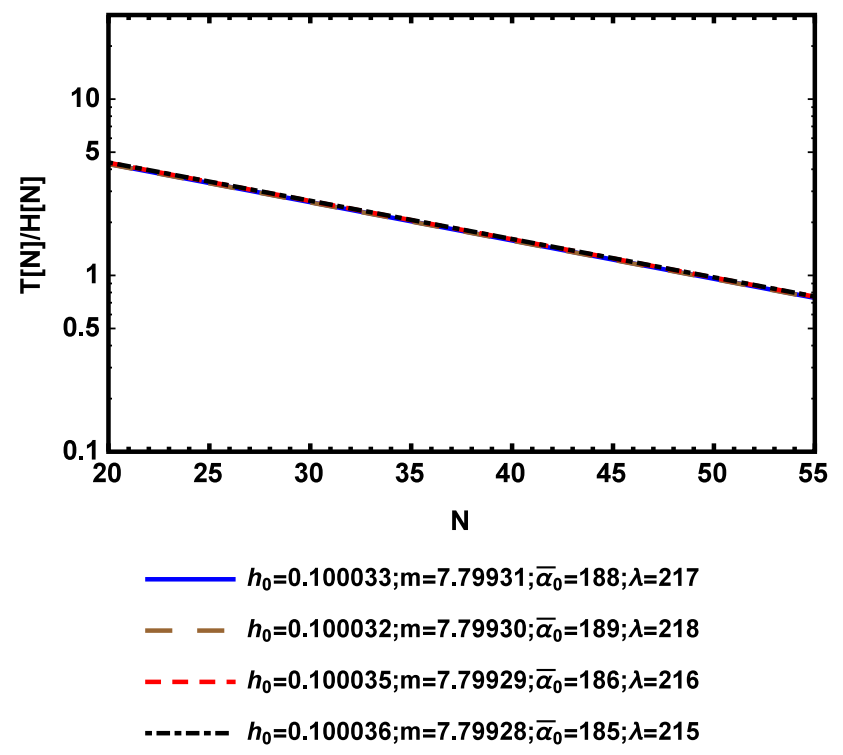

Fig. 6 The ratio of the temperature to the Hubble parameter during the inflationary evolution of the non-comoving model in the strong regime with power-law scale factors versus the number of e-folds for different values of the parameters of the model

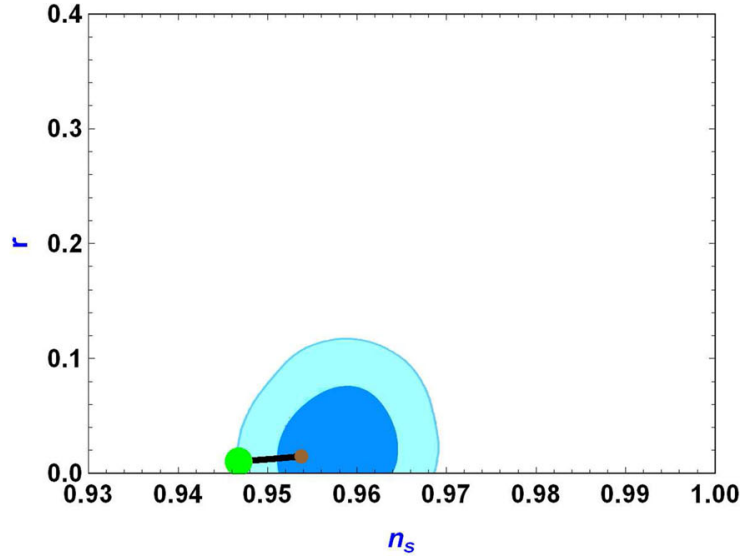

blue contours. In the right panel, the results of Planck 2018 are indicated by dark and light blue colors corresponding to $68 \%$ and $95 \%$ confidence levels, respectively. In both figures the thick black lines refer to the theoretical predictions, with the small, brown, and large, green, circles corresponding to the values of $n_{\mathrm{s}}$ at the number of e-folds $\mathcal{N}=50$ and $\mathcal{N}=55$, respectively

Consequently, by means of Eqs. (96) and (105), the scalar field at the horizon exit for the strong dissipative regime has the value

$\phi_{\star}=\phi_{e} \times \exp \left[-h_{0} \mathcal{N}\right]$.

Now we can calculate the scalar spectral index and the tensor to scalar ratio as given by Eqs. (123) and (129), respectively. The range of the acceptable values of the free parameters of the non-comoving warm inflationary model in the strong regime is presented in Fig. 5. In addition, to test the warm inflationary constraint that guaranties thermal fluctuations' dominance against quantum fluctuations, we plot the $T / H$ function in Fig. 6. Hence we see that the condition $T / H>1$ is satisfied for the adopted range of the model parameters. To obtain these results we have fixed the power spectrum based on its horizon exit value, i.e. $\mathcal{P}_{s}=2.17 \times 10^{-9}$, and we have used $\zeta=3, a_{\zeta}=0.0185$, and $b_{\zeta}=2.315$ in the definition of $G(Q)$. 
Table 4 Estimation of the free parameters of the non-comoving warm inflationary model with generalized de Sitter scale factors by using the Planck 2013, 2015 and 2018 data sets, and with $C_{\gamma}=55$, and $n=1$, respectively. We have restricted our analysis to real values of the scalar field at the horizon exit. To obtain the presented results we have fixed the power spectrum to its horizon exit value, i.e. $\mathcal{P}_{s}=2.17 \times 10^{-9}$, and we have used the numerical values $a_{\zeta}=0.0185$, and $b_{\zeta}=2.315$, respectively

\begin{tabular}{lllllllllll}
\hline$\gamma$ & $-\lambda$ & $\phi_{\star}$ & $\phi_{\mathrm{e}}$ & $h_{0}$ & $\alpha_{0}$ & $n_{\mathrm{s}}$ & $r_{\star} \times 10^{-17}$ & $-\mathcal{P}_{s 0} \times 10^{-22}$ & $T\left(\phi_{\star}\right) / H\left(\phi_{\star}\right)$ & $\mathcal{N}$ \\
\hline 4.20 & 18.890 & 0.000143554 & 0.00525699 & 0.06001 & 27.5 & 0.950937 & 6.3548 & 9.66527 & 1.11294 \\
4.25 & 18.895 & 0.000118045 & 0.00432542 & 0.06002 & 28 & 0.952282 & 2.93027 & 5.32686 & 1.09665 \\
4.30 & 18.897 & 0.000119332 & 0.00437521 & 0.06003 & 28.2 & 0.953098 & 3.06041 & 5.592 & 60 \\
4.35 & 18.899 & 0.000113554 & 0.00416586 & 0.06004 & 28.5 & 0.954025 & 2.51636 & 4.85727 & 1.0807 & 60 \\
\hline
\end{tabular}

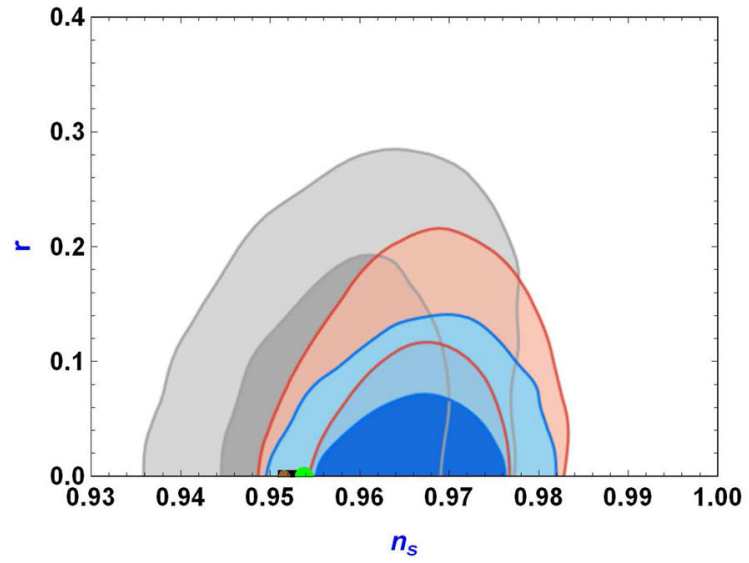

Fig. 7 The $r-n_{s}$ diagram comparing the predictions of the noncomoving warm inflation model in the strong dissipative regime with generalized de Sitter scale factors having the free parameters of Table 4, with the Planck 2013, 2015 and 2018 data sets. In the left panel, the likelihood of Planck 2013 are indicated with gray contours, Planck TT+lowP with red contours, and Planck TT,TE,EE+lowP (2015) with

\subsubsection{Strong dissipative regime with generalized de Sitter scale factor}

Now let us turn our attention to the generalized de Sitter scale factors model for the strong regime. Following the results of the Sect. 4.3, and the procedure of Sect. 4.3.1, we are going to examine the theoretical predictions for the strong dissipative regime of the non-comoving warm inflation with the generalized de Sitter scale factors, and compare them with the observational data sets obtained by the Planck satellite. In order to perform such a comparison, by using Eq. (124) to obtain the end of inflation values of the scalar field, we find the results presented in Table 4. Consequently, by using Eqs. (95) and (105), the scalar field at the horizon exit for the strong regime is expressed as

$\phi_{\star}=\phi_{e} \times \exp \left[-h_{0} \mathcal{N}\right]$.

By substituting Eq. (117) into Eqs. (85) and (95), for the strong regime with generalized de Sitter scale factors the scalar field potential as a function of the scalar field can be

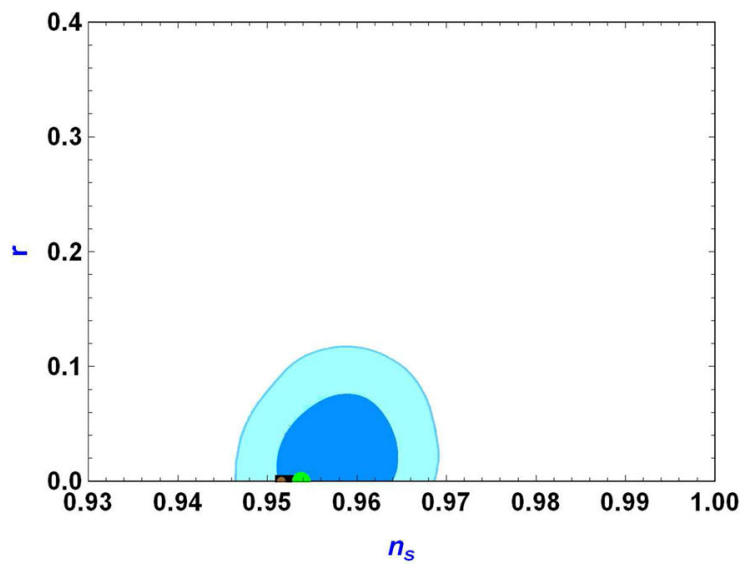

blue contours. In the right panel, the results of Planck 2018 are indicated by dark and light blue colors corresponding to $68 \%$ and $95 \%$ confidence levels, respectively. In both figures the thick black lines refer to the theoretical predictions, with the small, brown, and large, green, circles corresponding to the values of $n_{\mathrm{s}}$ at the number of e-folds $\mathcal{N}=55$ and $\mathcal{N}=65$, respectively

expressed as

$U(\phi)=4 \gamma(-\lambda+1)\left[\gamma^{2} h_{0}-(\lambda+2) \ln \left(-\frac{\lambda^{2}+\lambda-2}{\alpha_{0}^{2} \gamma h_{0}^{2} \phi^{2}}\right)\right]$.

Also from Eqs. (134) and (86), for the dissipation ratio $Q$ one obtains

$Q(\phi)=\frac{8\left(\lambda^{2}+\lambda-2\right)}{3 \gamma \phi\left(\gamma h_{0}^{2} \phi^{2}-8 \lambda(\lambda+1)+16\right)}$.

To find acceptable values of the free parameters of the model for the strong regime, one can consider the scalar spectral index and tensor-to-scalar ratio given by Eq. (123) and (129), respectively. The comparison of these quantities with the observational data is presented in Fig. 7.

In addition, we test the warm inflation constraint, which guarantees the thermal fluctuations' dominance over the quantum fluctuations. To do this we plot the function $T / H$, and we observe that the $T / H>1$ constraint is satisfied, 


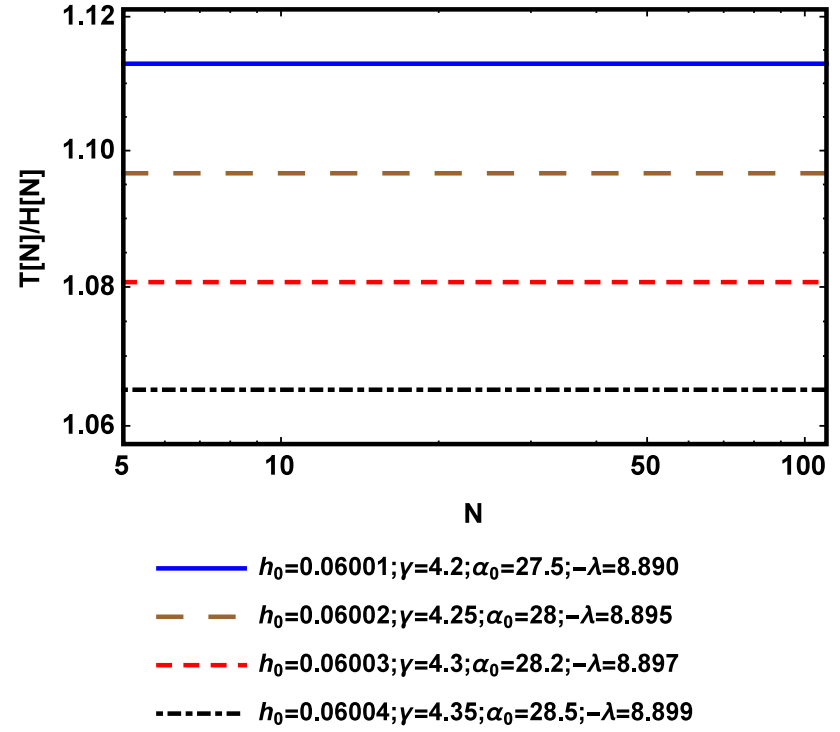

Fig. 8 The ratio of the temperature to the Hubble parameter during the inflationary evolution of the non-comoving warm inflationary model in the strong regime with generalized de Sitter scale factors, with $n=$ 1 , versus the number of e-folds, for different numerical values of the parameters of the model

as shown in Fig. 8. To obtain these results we have fixed the power spectrum based on its horizon exit value, i.e., $\mathcal{P}_{s}=2.17 \times 10^{-9}$, and we have used the numerical values $\zeta=3, a_{\zeta}=0.0185$, and $b_{\zeta}=2.315$ in the definition of $G(Q)$.

\section{Discussions and final remarks}

The assumption of the comoving motion of all the matter/energy components of the Universe is one of the cornerstones of present day cosmology. This mathematical choice of a universal reference frame allows a simple, but powerful theoretical description of the cosmological models to be given, and it directly leads to the Friedmann equations of standard cosmology, to the $\Lambda \mathrm{CDM}$ model, and to the inflationary paradigm, all formulated in the comoving frame. But, despite its remarkable success, still one may ask if from a physical point of view the assumption of the existence of a universal frame is really justified, especially if one takes into account the very different nature of the major components of the Universe, dark energy, dark and baryonic matter. If this is indeed the case, certainly a significant amount of fine tuning would be necessary to establish the universal cosmological frame. Alternatively, one may also assume that some specific physical processes in the early Universe led to the vanishing of all forms of anisotropy, and of the possible differences in the matter and energy components.
In the present paper, we have investigated the theoretical possibility that in the very early Universe, composed of a mixture of two basic constituents, namely, scalar field and radiation, the matter and field components have different fourvelocities. More exactly, we have considered this possibility in the framework of the warm inflationary model, in which matter, in the form of radiation, is generated during the initial cosmological expansion due to the decay of the scalar field. The warm inflationary scenario does not require a reheating phase at the end of inflation. Our basic assumption, and starting point in our analysis, is that the four-velocities of the radiation and scalar field are different, and thus they are not comoving. Such an interpretation can also be supported by a simple physical argument related to the decay of particles. Let us assume that a particle of mass $M$ decays into two particles with masses $m_{1}$ and $m_{2}$. The law of conservation of energy, as applied to the system of reference in which the particle is at rest, gives $M=E_{10}+E_{20}$, where $E_{10}$ and $E_{20}$ are the energies of the emerging particles. Then, by taking into account the energy conservation relation $E_{10}^{2}-m_{1}^{2}=E_{20}^{2}-m_{2}^{2}$, one can easily obtain the energies of the decay products as $E_{10}=$ $\left(M^{2}+m_{1}^{2}-m_{2}^{2}\right) / 2 M$, and $E_{20}=\left(M^{2}-m_{1}^{2}+m_{2}^{2}\right) / 2 M$, and obviously $E_{10} \neq E_{20} \neq M$. Consequently, the decay products will move with different four-velocities with respect to the particle generating them.

From a theoretical point of view the cosmological model with non-comoving scalar field and radiation is formally equivalent to a Universe model containing a single anisotropic fluid, having different pressure components along the coordinate axes. Moreover, the equivalent thermodynamic parameters of the anisotropic system are functions of the fourvelocities of the two components, and of their energy densities and pressures. As a first step in our analysis we have explicitly obtained the thermodynamic parameters of the anisotropic mixture of radiation and scalar field. Since the resulting single fluid description leads to an anisotropic matter/energy distribution, the resulting geometry is also anisotropic. We have explicitly obtained the gravitational field equations describing the non-comoving scalar field and dark radiation mixture for the simplest anisotropic Universe, described by a Bianchi Type I geometry.

We have implemented the basic idea of the warm inflation by splitting the total energy conservation equation into two components, describing the decay of the scalar field, and radiation creation, respectively. This splitting can be done naturally, without imposing any arbitrary form of the decay and creation rates, with $\Gamma_{\phi}$ and $\Gamma_{\text {rad }}$ determined by a function $F$ that depends on the differences in the four-velocities of the scalar field and radiation, and on the energy densities and pressures of the components. In building the theoretical model we have assumed that the difference in the four-velocities of the scalar field and radiation is small, and therefore one can also consider the deviations from isotropy 
as small, representing just a small perturbation of the homogeneous and isotropic background metric. But even if there is only a slight difference between the four-velocities of the scalar field and radiation, the early Universe would acquire some anisotropic characteristics, and its geometry will deviate from the standard FLRW one. This aspect already appears in Eq. (76), describing the radiation generation from the scalar field, and which contains the anisotropic radiation creation term $2 \alpha^{2} \dot{\phi}^{2} H_{3}$, which indicates that the distribution of the newly produced radiation is anisotropically distributed along the coordinate axis.

One of the essential tests of any physical model is its comparison with observations. In order to compare the noncomoving warm inflationary model with the Planck data we have adopted the standard slow-roll approximation, which is commonly used in inflationary models. In the formalism of the slow-roll inflation an essential quantity is the dissipation function, which can be represented as a function of $\alpha, \dot{\alpha}$, and $H$, where $\alpha$ is the rotation angle in the velocity space. When $\alpha=0$, the four-velocities of the scalar field and of the radiation coincide. In the present approach we have assumed that $\alpha$ is a decreasing function of time, with $\alpha \propto t^{-h_{0} m}$, and $\alpha \propto e^{-h_{0} \gamma t}$, respectively. The parameters $h_{0}$ and $m$ can be determined from the observational data, and this would also fix the transition towards a universal comoving frame of the warm inflationary model. For example, in the weak dissipation regime, $m \approx 30$, and $h_{0} \approx 0.01$ (see Table 1, which would give a decay law of $\alpha$ of the form $\alpha \propto t^{-0.3}$, which indicates just a slow transition towards isotropy. However, in the case of the generalized de Sitter expansion the decay of $\alpha$ is of the form $\alpha \propto e^{-3.3 t}$ (see Table 2), which would guarantee that the Universe enters in an isotropic and comoving phase at the end of inflation. In the case of the strong dissipative regime $\alpha \propto t^{-0.8}$ (Table 3) and $\alpha \propto e^{-0.24 t}$ (Table 4), respectively. Hence, in the noncomoving warm inflationary model with model parameters consistent with the Planck data, the possibility that a residual anisotropy from the early Universe did survive due to the non-comoving character of the scalar field and radiation fluid evolution cannot be excluded a priori.

The present analysis based on the slow-roll approximation also allow us to fix the functional form of the scalar field by essentially using the theoretical model, as well as the observations. Therefore there is no need to postulate in advance different forms of the scalar field potential. In the case of the strong dissipative regime with power-law scale factor the potential is also of power-law form, $U(\phi) \propto \phi^{-2 / h_{0} m}$, while in the other considered cases the potential is of a nonstandard form, involving quadratic and logarithmic terms. Finding the interpretation of these potentials from the elementary particle physics perspective is a matter of further research.

Further constraints on the non-comoving warm inflationary model can be obtained by considering the imprints of the non-comoving expansion on the Cosmic Microwave Background. The photons generated at the end of inflation at cosmological distances move in the Bianchi Type I Universe, having a geometry remnant from the non-comoving motion in the early Universe, along the geodesic lines, given by

$\frac{\mathrm{d} u^{\mu}}{\mathrm{d} \lambda}+\Gamma_{\alpha \beta}^{\mu} u^{\alpha} u^{\beta}=0$,

where $\lambda$ is an affine parameter, and the Christoffel symbols $\Gamma_{\alpha \beta}^{\mu}$ are obtained from the metric Eq. (60), and they are given by $\Gamma_{i i}^{0}=a_{i}^{2} H_{i}$, and $\Gamma_{0 i}^{i}=H_{i}, i=1,2,3$, respectively (no summation upon $i$ in the Christoffel symbols). As for the four-velocity $u^{\mu}=\mathrm{d} x^{\mu} / \mathrm{d} \lambda$ of the photons, it satisfies the normalization condition $u^{\mu} u_{\mu}=0$, giving $\left(u_{0}\right)^{2}=a_{i}^{2}\left(u^{i}\right)^{2}$. The temperature distribution is determined by the relation

$T(\hat{\mathbf{u}})=\frac{T_{*}}{1+z(\hat{\mathbf{u}})}$,

where the redshift $z$ is defined as $1+z\left(\lambda_{e}\right)=\tau\left(\lambda_{r}\right) / \tau\left(\lambda_{e}\right)$, where $\tau\left(\lambda_{r}\right)$ is the time difference of the received signals, and the present day values of the photon four-velocity are denoted as $u^{i}\left(t_{0}\right)=\hat{u}^{i}, i=1,2,3 . T_{*}$ denotes the last scattering temperature, which does not depend on the direction. But due the possible presence of anisotropies in the geometry of the Universe, photons traveling from different directions will be redshifted by different amounts. Therefore in an anisotropic Universe one must consider the spatial average $\bar{T}$ of the temperature field, which is given by $4 \pi \bar{T}=\int T(\hat{\mathbf{u}}) d \Omega_{\hat{\mathbf{u}}}$. Hence the anisotropies in the temperature field are given by $\delta T(\hat{\mathbf{u}})=1-T(\hat{\mathbf{u}}) / \bar{T}$. Another important observational parameter that could be used to test the anisotropy survival from the early non-comoving evolution is the multipole spectrum $Q_{l}$, which is obtained by considering the coefficients in the spherical expansion of the anisotropic temperature field. The quadrupole $Q_{2}$ is defined as $[144,176]$

$Q_{2}=\frac{2}{5 \sqrt{3}} \sqrt{e_{z}^{4}+e_{y}^{4}-e_{z}^{2} e_{y}^{2}}=\frac{2}{5 \sqrt{3}} e_{z}^{2}$,

where the eccentricities $e_{y}^{2}$ and $e_{z}^{2}$ are defined as $e_{y}^{2}=$ $\left(a_{1} / a_{2}\right)^{2}-1$ and $e_{z}^{2}=\left(a_{1} / a_{3}\right)^{2}-1$, respectively [176].

By using the nominal value of the best-fit quadrupole measured by Planck one can also constrain the parameters of the non-comoving warm inflationary model. Another independent test of the model could be provided by the observations of the anisotropies in the expansion rates of the Universe, which would determine luminosity distance-redshift relationships that are non-invariant rotationally [176]. Such relationships obtained from the study of the Ia type supernovae can be therefore used to constrain the non-comoving warm inflationary models, and to identify their imprints on the anisotropies present in the Universe. 
There is some observational evidence, as shown by the recent Planck data [1-6], that suggests that even though on the large scale the Universe is homogeneous, small deviations from isotropy may still be present on the cosmological scales. In the present paper we have proposed a model that may account for the presence of these anisotropies as generated during the warm inflationary evolution in the very early Universe. One of the basic postulates of present day cosmology is the possibility of choosing a comoving reference frame for all components of the Universe, no matter their nature. However, there is no general principle requiring that such a frame must have existed at all times in the Universe, and therefore in our present investigation we have assumed that the scalar field generating the radiation in the very early Universe did in fact have a different four-velocity as compared to those of the photons. Hence a comoving frame could have been established dynamically, due to the time decay of the angle $\alpha$ describing the differences between the four-velocities of the two major initial components of the Universe. The possibility of non-comoving cosmological motions, their impact on the evolution of the Universe and some observational implications will be considered in a future publication.

Acknowledgements H. S. thanks H. Firouzjahi for constructive discussions on inflation and perturbations.

Data Availability Statement This manuscript has no associated data or the data will not be deposited. [Authors' comment: The datasets generated during and/or analyzed during the current study are available from the corresponding author on reasonable request.]

Open Access This article is licensed under a Creative Commons Attribution 4.0 International License, which permits use, sharing, adaptation, distribution and reproduction in any medium or format, as long as you give appropriate credit to the original author(s) and the source, provide a link to the Creative Commons licence, and indicate if changes were made. The images or other third party material in this article are included in the article's Creative Commons licence, unless indicated otherwise in a credit line to the material. If material is not included in the article's Creative Commons licence and your intended use is not permitted by statutory regulation or exceeds the permitted use, you will need to obtain permission directly from the copyright holder. To view a copy of this licence, visit http://creativecomm ons.org/licenses/by/4.0/.

Funded by SCOAP ${ }^{3}$.

\section{References}

1. P.A.R. Ade et al., Planck 2013 results. I. Astron. Astrophys. 571, A1 (2014). arXiv:1303.5062 [astro-ph.CO]

2. P.A.R. Ade et al., Planck 2013 results. XVI. Astron. Astrophys. 571, A16 (2014). arXiv:1303.5076 [astro-ph.CO]

3. P.A.R. Ade et al., Planck 2013 results. XXII. Astron. Astrophys. 571, A22 (2014). arXiv:1303.5082 [astro-ph.CO]

4. P.A.R. Ade et al., Planck 2013 results. XXIV. Astron. Astrophys. 571, A24 (2014). arXiv: 1303.5084 [astro-ph.CO]

5. P.A.R. Ade et al., Planck 2013 results. XXVI. Astron. Astrophys. 571, A26 (2014). arXiv:1303.5086 [astro-ph.CO]
6. Y. Akrami et al. (Planck Collaboration), Planck 2018 results. X. Constraints on inflation. Astron. Astrophys. 641, A10 (2020). arXiv:1807.06211 [astro-ph.CO]

7. N. Aghanim et al. (Planck Collaboration), Planck 2018 results. VI. Cosmological parameters . Astron. Astrophys. 641, A6 (2020). arXiv: 1807.06209 [astro-ph.CO]

8. P.J.E. Peebles, B. Ratra, Rev. Mod. Phys. 75, 559 (2003)

9. T. Padmanabhan, Phys. Rep. 380, 235 (2003)

10. A.G. Riess et al., Astron. J. 116, 1009 (1998)

11. S. Perlmutter et al., Astrophys. J. 517, 565 (1999)

12. P. de Bernardis et al., Nature 404, 955 (2000)

13. S. Hanany et al., Astrophys. J. 545, L5 (2000)

14. S. Weinberg, Rev. Mod. Phys. 61, 1 (1989)

15. M. Li, X.-D. Li, S. Wang, Y. Wang, Front. Phys. 8, 828 (2013)

16. H. Sheikhahmadi, A. Aghamohammadi, K. Saaidi, Adv. High Energy Phys. 2016, 2594189 (2016)

17. K. Arun, S.B. Gudennavar, C. Sivaram, Adv. Space Res. 60, 166 (2017)

18. P. Brax, Rep. Prog. Phys. 81, 016902 (2018)

19. N. Frusciante, L. Perenon, Phys. Rep. 857, 1 (2020)

20. C. Wetterich, Nucl. Phys. B 302, 645 (1988)

21. P.J.E. Peebles, B. Ratra, Astrophys. J. Lett. 325, L17 (1988)

22. B. Ratra, P.J.E. Peebles, Phys. Rev. D 37, 3406 (1988)

23. R.R. Caldwell, R. Dave, P.J. Steinhardt, Phys. Rev. Lett. 80, 1582 (1998)

24. L. Amendola, Phys. Rev. D 62, 043511 (2000)

25. C. Armendariz-Picon, V.F. Mukhanov, P.J. Steinhardt, Phys. Rev. Lett. 85, 4438 (2000)

26. T. Chiba, T. Okabe, M. Yamaguchi, Phys. Rev. D 62, 023511 (2000)

27. C. Armendariz-Picon, V.F. Mukhanov, P.J. Steinhardt, Phys. Rev. D 63, 103510 (2001)

28. A. Sen, JHEP 0207, 065 (2002)

29. A. Mohammadi, T. Golanbari, H. Sheikhahmadi, K. Sayar, L. Akhtari, M. Rasheed, K. Saaidi, Chin. Phys. C 44(9), 095101 (2020). arXiv:2001.10042 [gr-qc]

30. R.R. Caldwell, Phys. Lett. B 545, 23 (2002)

31. R.R. Caldwell, M. Kamionkowski, N.N. Weinberg, Phys. Rev. Lett. 91, 071301 (2003)

32. J.M. Cline, S.Y. Jeon, G.D. Moore, Phys. Rev. D 70, 043543 (2004)

33. E. Elizalde, S. Nojiri, S.D. Odinstov, Phys. Rev. D 70, 043539 (2004)

34. S. Nojiri, S.D. Odintsov, S. Tsujikawa, Phys. Rev. D 71, 063004 (2005)

35. A. Anisimov, E. Babichev, A. Vikman, J. Cosmol. Astropart. Phys. 06, $006(2005)$

36. D.F. Mota, J.D. Barrow, Phys. Lett. B 581, 141 (2004)

37. J. Khoury, A. Weltman, Phys. Rev. Lett. 93, 171104 (2004)

38. J. Khoury, A. Weltman, Phys. Rev. D 69, 044026 (2004)

39. Kh Saaidi, A. Mohammadi, H. Sheikhahmadi, Phys. Rev. D 83, 104019 (2011)

40. Kh Saaidi, A. Mohammadi, Phys. Rev. D 85, 023526 (2012)

41. Kh Saaidi, A. Mohammadi, T. Golanbari, H. Sheikhahmadi, B. Ratra, Phys. Rev. D 86, 045007 (2012)

42. AYu. Kamenshchik, U. Moschellai, V. Pasquier, Phys. Lett. B 511, $265(2001)$

43. M.C. Bento, O. Bertolami, A.A. Sen, Phys. Rev. D 66, 043507 (2002)

44. O. Bertolami, C.G. Boehmer, T. Harko, F.S.N. Lobo, Phys. Rev. D 75, 104016 (2007)

45. T. Harko, F.S.N. Lobo, Eur. Phys. J. C 70, 373 (2010)

46. T.P. Sotiriou, V. Faraoni, Rev. Mod. Phys. 82, 451 (2010)

47. A. De Felice, S. Tsujikawa, Living Rev. Relativ. 13, 3 (2010)

48. S. Nojiri, S.D. Odintsov, Phys. Rep. 505, 59 (2011) 
49. T. Harko, F.S.N. Lobo, S. Nojiri, S.D. Odintsov, Phys. Rev. D 84, 024020 (2011)

50. T. Harko, T.S. Koivisto, F.S.N. Lobo, G.J. Olmo, Phys. Rev. D 85, 084016 (2012)

51. T. Harko, F.S.N. Lobo, Extensions of $f(R)$ Gravity: CurvatureMatter Couplings and Hybrid MetricPalatini Theory. Cambridge Monographs on Mathematical Physics (Cambridge University Press, Cambridge, 2018)

52. J.M. Overduin, P.S. Wesson, Phys. Rep. 402, 267 (2004)

53. P. Morley, D. Buettner, Universe 3, 81 (2017)

54. L.E. Strigari, Rep. Prog. Phys. 81, 056901 (2018)

55. L.S. Kisslinger, D. Das, Int. J. Mod. Phys. A 34, 1930013 (2019)

56. A. Guth, Phys. Rev. D 23, 347 (1981)

57. V. Mukhanov, Physical Foundations of Cosmology (Cambridge University Press, Cambridge, 2005)

58. A. Linde, Phys. Lett. B 108, 389 (1982)

59. A. Albrecht, P.J. Steinhardt, Phys. Rev. Lett. 48, 1220 (1982)

60. A. Linde, Phys. Lett. B 129, 177 (1983)

61. A. Linde, Phys. Rev. D 49, 748 (1994)

62. R.H. Cyburt, B.D. Fields, K.A. Olive, T.-H. Yeh, Rev. Mod. Phys. 88, 015004 (2016)

63. S. Nojiri, S.D. Odintsov, V.K. Oikonomou, Phys. Rep. 692, 1 (2017)

64. A. Albrecht, P.J. Steinhardt, M.S. Turner, F. Wilczek, Phys. Rev. Lett. 48, 1437 (1982)

65. L. Kofman, A. Linde, A.A. Starobinsky, Phys. Rev. Lett. 73, 3195 (1994)

66. L. Kofman, A. Linde, A.A. Starobinsky, Phys. Rev. D 56, 3258 (1997)

67. T. Harko, W.F. Choi, K.C. Wong, K.S. Cheng, J. Cosmol. Astropart. Phys. 06, 002 (2008)

68. R.K. Jain, P. Chingangbam, L. Sriramkumar, Nucl. Phys. B 852, $366(2011)$

69. V. Demozzi, C. Ringeval, J. Cosmol. Astropart. Phys. 05, 009 (2012)

70. H. Motohashi, A. Nishizawa, Phys. Rev. D 86, 083514 (2012)

71. K. Mukaida, K. Nakayama, J. Cosmol. Astropart. Phys. 03, 002 (2013)

72. A. Nishizawa, H. Motohashi, Phys. Rev. D 89, 063541 (2014)

73. L. Dai, M. Kamionkowski, J. Wang, Phys. Rev. Lett. 113, 041302 (2014)

74. J. Martin, C. Ringeval, V. Vennin, Phys. Rev. Lett. 114, 081303 (2015)

75. C. Gross, O. Lebedev, M. Zatta, Phys. Lett. B 753, 178 (2016)

76. Y.S. Myung, T. Moon, J. Cosmol. Astropart. Phys. 07, 014 (2016)

77. M. Rinaldi, L. Vanzo, Phys. Rev. D 94, 024009 (2016)

78. T. Rehagen, G.B. Gelmini, J. Cosmol. Astropart. Phys. 06, 039 (2015)

79. R. Allahverdi, R. Brandenberger, F. Cyr-Racine, A. Mazumdar, Annu. Rev. Nucl. Part. Sci. 60, 27 (2010)

80. M.A. Amin, M.P. Hertzberg, D.I. Kaiser, J. Karouby, Int. J. Mod. Phys. D 24, 1530003 (2015)

81. A. Berera, L.-Z. Fang, Phys. Rev. Lett. 74, 1912 (1995)

82. A. Berera, Phys. Rev. Lett. 75, 3218 (1995)

83. A. Berera, Phys. Rev. D 54, 2519 (1996)

84. A. Berera, Phys. Rev. D 55, 3346 (1997)

85. A. Berera, M. Gleiser, R.O. Ramos, Phys. Rev. D 58, 123508 (1998)

86. A. Berera, T.W. Kephart, Phys. Rev. Lett. 83, 1084 (1999)

87. A. Berera, Nucl. Phys. B 585, 666 (2000)

88. A. Berera, M. Gleiser, R.O. Ramos, Phys. Rev. Lett. 83, 264 (1999)

89. H.P. De Oliveira, S.E. Jorás, Phys. Rev. D 64, 063513 (2001)

90. L.M.H. Hall, I.G. Moss, A. Berera, Phys. Rev. D 69, 083525 (2004)

91. I.G. Moss, C.M. Graham, Phys. Rev. D 78, 123526 (2008)

92. Y. Zhang, JCAP 03, 023 (2009)
93. N. Barnaby, Z. Huang, Phys. Rev. D 80, 126018 (2009)

94. M. Bastero-Gil, A. Berera, R.O. Ramos, J. Cosmol. Astropart. Phys. 1107, 030 (2011)

95. R. Herrera, M. Olivares, N. Videla, Phys. Rev. D 88, 063535 (2013)

96. M.R. Setare, V. Kamali, Phys. Lett. B 726, 56 (2013)

97. R.O. Ramos, L.A. da Silva, J. Cosmol. Astropart. Phys. 1303, 032 (2013)

98. X.-M. Zhang, J.-Y. Zhu, JCAP 02, 005 (2014)

99. M. Bastero-Gil, A. Berera, R.O. Ramos, J.G. Rosa, JCAP 1410, 053 (2014)

100. G. Piccinelli, A. Sanchez, A. Ayala, A.J. Mizher, Phys. Rev. D 90, 083504 (2014)

101. X.-M. Zhang, J.-Y. Zhu, Phys. Rev. D 91, 063510 (2015)

102. G. Panotopoulos, N. Videla, Eur. Phys. J. C 75, 525 (2015)

103. M. Bastero-Gil, A. Berera, R.-O. Ramos, J.G. Rosa, Phys. Rev. Lett. 117, 151301 (2016)

104. M. Motaharfar, H.-R. Sepangi, Eur. Phys. J. C 76, 646 (2016)

105. K. Sayar, A. Mohammadi, L. Akhtari, K. Saaidi, Phys. Rev. D 95, 023501 (2017)

106. M. Benetti, R.-O. Ramos, Phys. Rev. D 95, 023517 (2017)

107. A. Jawad, N. Videla, F. Gulshan, Eur. Phys. J. C 77, 271 (2017)

108. M. Motaharfar, E. Massaeli, H.-R. Sepangi, Phys. Rev. D 96, 103541 (2017)

109. A. Jawad, S. Chaudhary, N. Videla, Eur. Phys. J. C 77, 808 (2017)

110. A. Jawad, S. Hussain, S. Rani, N. Videla, Eur. Phys. J. C 77, 700 (2017)

111. P. Goodarzi, H.-M. Sadjadi, Eur. Phys. J. C 77, 463 (2017)

112. R. Herrera, J. Cosmol. Astropart. Phys. 5, 029 (2017)

113. K. Li, X.-M. Zhang, H.-Y. Ma, J.-Y. Zhu, Phys. Rev. D 98, 123528 (2018)

114. V. Kamali, Eur. Phys. J. C 78, 975 (2018)

115. M. Bastero-Gil, A. Berera, R. Hernández-Jiménez, J.-G. Rosa, Phys. Rev. D 98, 083502 (2018)

116. M. Motaharfar, E. Massaeli, H.-R. Sepangi, J. Cosmol. Astropart. Phys. 10, 002 (2018)

117. X.-B. Li, Y.-Y. Wang, H. Wang, J.-Y.Zhu, Phys. Rev. D 98, 043510 (2018)

118. L.-L. Graef, R.-O. Ramos, Phys. Rev. D 98, 023531 (2018)

119. A. Berera, J. Mabillard, M. Pieroni, R.-O. Ramos, J. Cosmol. Astropart. Phys. 7, 021 (2018)

120. N. Videla, G. Panotopoulos, Phys. Rev. D 97, 123503 (2018)

121. X. Tong, Y. Wang, S. Zhou, J. Cosmol. Astropart. Phys. 6, 013 (2018)

122. Z.-P. Peng, J.-N. Yu, X.-M. Zhang, J.-Y. Zhu, Phys. Rev. D 97, 063523 (2018)

123. X.-B. Li, H. Wang, J.-Y. Zhu, Phys. Rev. D 97, 063516 (2018)

124. Y.-Y. Wang, J.-Y. Zhu, X.-M. Zhang, Phys. Rev. D 97, 063510 (2018)

125. R. Herrera, Eur. Phys. J. C 78, 245 (2018)

126. M. Bastero-Gil, A. Berera, R.-O. Ramos, J.-G. Rosa, J. High Energy Phys. 1802, 063 (2018)

127. M. Bastero-Gil, S. Bhattacharya, K. Dutta, M.-R. Gangopadhyay, J. Cosmol. Astropart. Phys. 2, 054 (2018)

128. R. Arya, A. Dasgupta, G. Goswami, J. Prasad, R. Rangarajan, J. Cosmol. Astropart. Phys. 2, 043 (2018)

129. Y.-Y. Wang, J.-Y. Zhu, X.-M. Zhang, Phys. Rev. D 99, 103529 (2019)

130. M. Bastero-Gil, A. Berera, R. Hernández-Jiménez, J.-G. Rosa, Phys. Rev. D 99, 103520 (2019)

131. J.-G. Rosa, L.-B. Ventura, Phys. Rev. Lett. 122, 161301 (2019)

132. S. Das, Phys. Rev. D 99, 063514 (2019)

133. M. Motaharfar, V. Kamali, R.-O. Ramos, Phys. Rev. D 99, 063513 (2019)

134. X.-B. Li, X.-G. Zheng, J.-Y. Zhu, Phys. Rev. D 99, 043528 (2019) 
135. S. Rasouli, K. Rezazadeh, A. Abdolmaleki, K. Karami, Eur. Phys. J. C 79, 79 (2019)

136. K. Dimopoulos, L. Donaldson-Wood, Phys. Lett. B 796, 26 (2019)

137. H. Sheikhahmadi, A. Mohammadi, A. Aghamohammadi, T. Harko, R. Herrera, C. Corda, A. Abebe, K. Saaidi, Eur. Phys. J. C 79, 1038 (2019)

138. T. Harko, H. Sheikhahmadi, Phys. Dark Univ. 28, 100521 (2020)

139. C.J. Copi, D. Huterer, D.J. Schwarz, G.D. Starkman, Adv. Astron. 2010, 847541 (2010)

140. K. Migkas, G. Schellenberger, T.H. Reiprich, F. Pacaud, M.E. Ramos-Ceja, L. Lovisari, Astron. Astrophys. 636, A15 (2020)

141. E.O. Zavarygin, J.K. Webb, Mon. Not. R. Astron. Soc. 489, 3966 (2019)

142. T.S. Koivisto, D.F. Mota, M. Quartin, T.G. Zlosnik, Phys. Rev. D 83, 023509 (2011)

143. M. Watanabe, S. Kanno, J. Soda, MNRAS Lett. 412(2011), L83 (2011)

144. J. Soda, Class. Quantum Gravity 29, 083001 (2012)

145. P. Aluri, S. Panda, M. Sharma, S. Thakur, JCAP 12, 003 (2013)

146. N. Bartolo, S. Matarrese, M. Peloso, A. Ricciardone, Phys. Rev. D 87, 023504 (2013)

147. Z. Chang, S. Wang, Eur. Phys. J. C 73, 2516 (2013)

148. S.A. Appleby, E.V. Linder, Phys. Rev. D 87, 023532 (2013)

149. J.B. Jimenez, V. Salzano, R. Lazkoz, Phys. Lett. B 741, 168 (2015)

150. T.S. Pereira, G.A. Mena Marugán, S. Carneiro, JCAP 1507, 029 (2015)

151. P. Sundell, T. Koivisto, Phys. Rev. D 92, 123529 (2015)

152. T. Pailas, T. Christodoulakis, J. Cosmol. Astropart. Phys. 07, 029 (2019)

153. M. Thorsrud, B.D. Normann, T.S. Pereira, Class. Quantum Gravity $37,065015(2020)$

154. T. Harko, F.S.N. Lobo, JCAP 07, 036 (2013)

155. P.S. Letelier, Phys. Rev. D 22, 807 (1980)

156. P.S. Letelier, P.S.C. Alencar, Phys. Rev. D 34, 343 (1986)

157. S.R. Oliveira, Phys. Rev. D 40, 3976(1989)
158. L. Herrera, N.O. Santos, Phys. Rep. 286, 53 (1997)

159. J.A.R. Cembranos, A.L. Maroto, H. Villarrubia-Rojo, J. Cosmol. Astropart. Phys. 06, 041 (2019)

160. T. Harko, F.S.N. Lobo, Phys. Rev. D 83, 124051 (2011)

161. T. Harko, F.S.N. Lobo, Astropart. Phys. 35, 547 (2012)

162. A.L. Maroto, JCAP 0605, 015 (2006)

163. A.L. Maroto, Int. J. Mod. Phys. D 15, 2165 (2006)

164. J.B. Jimenez, A.L. Maroto, Phys. Rev. D 76, 023003 (2007)

165. J.B. Jimenez, A.L. Maroto, JCAP 0903, 015 (2009)

166. V. Faraoni, Phys. Rev. D 85, 024040 (2012)

167. C. Gergely, Z. Keresztes, L.Á. Gergely, Phys. Rev. D 102, 024044 (2020)

168. C. Bonvin, R. Durrer, M.A. Gasparini, Phys. Rev. D 73, 023523 (2006)

169. D.J. Schwarz, B. Weinhorst, Astron. Astrophys. 474, 717 (2007)

170. J.D. Barrow, A.R. Liddle, C. Pahud, Phys. Rev. D 74, 127305 (2006)

171. J.D. Barrow, A.R. Liddle, Phys. Rev. D 47, R5219 (1993)

172. M. Naderi, A. Aghamohammadi, A. Refaei, H. Sheikhahmadi, Mod. Phys. Lett. A 34, 1950272 (2019)

173. Z. Ghadiri, A. Refaei, A. Aghamohammadi, H. Sheikhahmadi, Mod. Phys. Lett. A 35, 2050078 (2020)

174. A. Mohammadi, K. Saaidi, H. Sheikhahmadi, Phys. Rev. D 100, 083520 (2019)

175. A. Mohammadi, T. Golanbari, H. Sheikhahmadi, K. Sayar, L. Akhtari, M.A. Rasheed, K. Saaidi, Chin. Phys. C 44, 095101 (2020)

176. T. Koivisto, D.F. Mota, JCAP 0806, 018 (2009) 